\title{
SIX1 Reprograms Myogenic Transcription Factors to Maintain the Rhabdomyosarcoma undifferentiated state
}

Jessica Y. Hsu',2, Etienne P. Danis ${ }^{1,10}$, Stephanie Nance ${ }^{8}$, Jenean O'Brien ${ }^{3}$, Veronica M. Wessells ${ }^{4}$, Andrew E. Goodspeed $^{1,10}$, Jared C. Talbot ${ }^{5}$, Sharon L. Amacher ${ }^{6}$, Paul Jedlicka ${ }^{7}$, Joshua C. Black ${ }^{1,2}$, James C. Costello ${ }^{1,2,10}$, Adam D. Durbin ${ }^{8}$, Kristin B. Artinger ${ }^{9,10^{*}}$, Heide L. Ford ${ }^{1,2,10^{*}}$

1. Department of Pharmacology, University of Colorado Anschutz Medical Campus, Aurora, CO, USA

2. Pharmacology Graduate Program, University of Colorado Anschutz Medical Campus, Aurora, CO, USA

3. Department of Biology, College of St. Scholastica, Duluth, MN, USA

4. Division of Medical Oncology, University of Colorado Anschutz Medical Campus, Aurora, CO, USA

5. School of Biology and Ecology, University of Maine, Orono, ME, USA

6. Department of Molecular Genetics, Ohio State University, Columbus, OH, USA

7. Department of Pathology, University of Colorado Anschutz Medical Campus, Aurora, CO, USA

8. Division of Molecular Oncology, St. Jude Children's Research Hospital, Memphis, TN, USA

9. Department of Craniofacial Biology, University of Colorado Anschutz Medical Campus, Aurora, CO, USA

10. University of Colorado Cancer Center, University of Colorado Anschutz Medical Campus, Aurora, CO, USA

*Correspondence: heide.ford@cuanschutz.edu, kristin.artinger@cuanschutz.edu

Keywords: Rhabdomyosarcoma, SIX1, MYOD1, muscle differentiation, muscle progenitor, transcriptional control

- SIX1 prevents differentiation in RMS while it promotes differentiation during normal development

- FN-RMS are highly dependent on SIX1 for growth in both zebrafish and mouse xenograft models

- Loss of SIX1 alters the transcriptional landscape of RMS cells, inducing a growth to differentiation switch

- SIX1 knockdown in FN-RMS causes reduced super enhancer-based activity at stem-related genes and enhanced MYOD1 binding to differentiation loci, resulting in the activation of a myogenic differentiation program

- A gene signature derived from SIX1 loss strongly correlates with myogenic differentiation status and is predictive of advanced RMS. 


\section{Summary}

34 Rhabdomyosarcoma (RMS) is a pediatric skeletal muscle sarcoma characterized by the expression of 35 the myogenic-lineage transcription factors (TF) MYOD1 and MYOG. Despite high expression of these 36 TFs, RMS cells fail to terminally differentiate, suggesting the presence of factors that alter their function. 37 Here, we demonstrate that the developmental TF, SIX1, is highly expressed in RMS and is critical to 38 maintain a muscle progenitor-like state. SIX1 loss induces terminal differentiation of RMS cells into 39 myotube-like cells and dramatically impedes tumor growth in vivo. We show that SIX1 maintains the RMS 40 undifferentiated state by controlling enhancer activity and MYOD1 occupancy at loci more permissive to 41 tumor growth over terminal muscle differentiation. Finally, we demonstrate that a gene signature derived 42 from SIX1 loss correlates with differentiation status in RMS and predicts RMS progression in human 43 disease. Our findings demonstrate a master regulatory role for SIX1 in the repression of RMS 44 differentiation via genome-wide alterations in MYOD1-mediated transcription.

45

46

47

48

49

50

51

52

53

54

55

56

57 


\section{Introduction}

Rhabdomyosarcoma (RMS) is a soft tissue pediatric sarcoma with molecular and histological features that resemble undifferentiated skeletal muscle. The majority of pediatric RMS cases can be divided into two major subtypes: Embryonal RMS (ERMS) and Alveolar RMS (ARMS), which are designated based on their histology. While ERMS tumors are characterized by a variety of mutational events, notably $R A S$ mutations, ARMS tumors are classically associated with PAX3-FOXO1 or PAX7-FOXO1 chromosomal rearrangements, which has led to the replacement of the histological annotations ERMS and ARMS with "Fusion-negative (FN)" and "Fusion-positive (FP)". The distinct genetic perturbations associated with ERMS and ARMS have long implied that the RMS subtypes arise from distinct mechanisms, however a shared feature of all RMS tumors is their expression of the myogenic regulatory transcription factors (TF) MYOD1 and MYOG, orchestrators of skeletal muscle differentiation with aberrant functions in RMS tumors $^{1}$. Whereas in normal skeletal muscle differentiation these myogenic TFs coordinate the expansion, commitment, and eventual differentiation of embryonic mesodermal or myogenic progenitors, the expression of these myogenic TFs in RMS tumors is not coupled with exit from the cell cycle and differentiation into post-mitotic myofibers ${ }^{2}$. Several studies to date have discovered distinct activities of these myogenic transcription factors in the context of normal muscle development and $\mathrm{RMS}^{3-5}$. However, it remains less clear what factors cause these myogenic regulatory factors to depart from their canonical roles as drivers of muscle differentiation to instead maintain RMS cells as less differentiated muscle progenitors.

The SIX1 homeodomain-containing TF belongs to the Six gene family that includes $S I X 1-S I X 6$ in vertebrates. Early studies of the SIX1 ortholog in drosophila, sine oculis (so), placed the functions of the Six gene family in eye morphogenesis, as so mutants lack compound eye structures ${ }^{6}$. However, since the original discovery of so, the functions of the Six family genes are known to extend beyond the visual system in vertebrates. Notably, the mammalian orthologs Six1 and Six4 have conserved and 
indispensable roles in embryonic skeletal muscle development and skeletal muscle regeneration. In mice, Six 1 deficiency alone causes reduced and disorganized muscle mass ${ }^{7}$, and further ablation of Six 1 and its ortholog Six4 causes exacerbated craniofacial defects and severe muscle hypoplasia ${ }^{8}$. In both Six 1 and Six1/Six4 deficient mouse models, the expression of the critical myogenic TFs MYOD1 and MYOG is compromised in migrating hypaxial muscle, demonstrating that Six 1 and Six4 are required for the activation of these myogenic TFs. In zebrafish, morpholino-mediated loss of six1b gene expression similarly causes reduced hypaxial muscle and impairment of $\operatorname{Pax} 7+$ muscle stem cell proliferation during skeletal muscle repair ${ }^{9,10}$. Recently, genetic ablation of six $1 a /$ six $1 b /$ six $4 a / s i x 4 b$ paralogs in the zebrafish genome has additionally shown that compound loss of six1/4 function causes complete loss of all migratory muscle precursors that generate hypaxial muscles such as the fin muscles, while leaving trunk muscle relatively unaffected ${ }^{11}$. These results align with previous observations that morpholino-mediated loss of six1a and six1b also affect hypaxial muscles, though the muscle defects observed in the

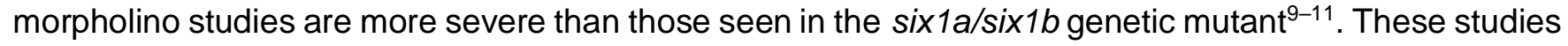
demonstrate that Six1, which acts in concert with Six4, lies upstream of the myogenic specification gene regulatory network and is a necessary component of the skeletal muscle circuit.

Myogenic differentiation is tightly governed by a cascade of myogenic regulatory factor (MRF) expression which encompass the highly conserved class II basic helix-loop-helix (bHLH) TFs MYOD1, MYF5, MYOG, and MRF4. During the course of embryonic development as well as skeletal muscle repair and regeneration, these four MRFs are considered necessary for committing progenitor cells to the skeletal muscle lineage, expanding the progenitor cell pool, and differentiating committed cells into contractile muscle fibers ${ }^{12}$. While structurally the MRF family is conserved, the transition of muscle progenitors from commitment, to growth, and subsequently to differentiation invokes sub-functionalized and contextspecific roles of these MRFs. Indeed, MyoD1 can activate distinct myoblast-specific and differentiationspecific gene expression programs by modifying chromatin environments that facilitate either differentiation or myoblast growth ${ }^{13,14}$. Because the functions of MYOD1 are co-opted in RMS tumors to 
109

110

foster growth rather than to promote differentiation, we hypothesized that other factors critical for normal skeletal muscle development must repress the differentiation subprograms of MYOD1. Given the wellestablished role of SIX1 in regulating upstream activities of MYOD1 as well as other MRFs to induce skeletal muscle development ${ }^{8,10,15-17}$, we investigated the molecular role of $S I X 1$ in regulating RMS tumor growth. Here, we report that SIX1 loss causes a growth-to-differentiation switch in RMS cells by globally regulating a myogenic transcriptional program and reinstating the function of MYOD1 as a driver of skeletal muscle differentiation.

\section{Results}

\section{SIX1 is overexpressed and predicted to be an essential gene in Rhabdomyosarcoma}

To examine whether SIX1 is highly expressed in human RMS, we interrogated its expression in publicly available large RMS RNAseq datasets. In multiple independent datasets, high SIX1 mRNA expression could be detected, both compared with other sarcomas in the National Cancer Institute Oncogenomics pan-sarcoma dataset (Suppl Fig. 1A) and the St. Jude Pediatric Cancer Genome Project (Suppl Fig. 1B), and compared with normal tissues in the St. Jude Integrated Rhabdomyosarcoma Database (iRDb) (Fig 1A). Notably, SIX1 was more highly expressed in RMS samples, compared with differentiated skeletal muscle controls depicting different stages of skeletal muscle development (Fig 1A). To confirm these data, we next assessed SIX1 protein expression in an RMS tumor tissue array consisting of 96 human RMS patient samples and 8 normal skeletal muscle controls (Figure 1B-C). Using a 1-4 scoring system of nuclear immunohistochemical staining, we detected strong nuclear SIX1 staining in the ERMS/FusionNegative and ARMS/Fusion-Positive tumor sections (18\% and $29 \%$ with $\mathrm{IHC}$ staining scores $\geq 2$, respectively) compared to normal skeletal muscle control sections $(0 \%$ with $\mathrm{IHC}$ staining score $\geq 2)$ (Figure 1B-C). To further determine if SIX1 has a functional role in RMS, we next examined data from the Broad and Sanger Institutes' exome-wide CRISPR-Cas9 knockout (KO) screening dataset ${ }^{18}$. In the 869 cell lines tested in the CRISPR-Cas9 screen, we observed that the 10 RMS cell lines used in the 
134

135

136

screen exhibited both high SIX1 mRNA expression and high SIX1 gene dependency (Figure 1D). Further comparison of the RMS tumor cell lines against all other tumor cell lines demonstrates that SIX1 is a selective dependency in RMS and is required for RMS cell survival ( $q$-value $=0.018$ ), as is the myogenic TF MYOD1 (Figure 1E).

Given the high expression of SIX1 in RMS tumors compared with matched normal tissues, we hypothesized that the increased SIX1 expression in RMS tumors compared with normal muscle could aberrantly activate its developmental functions in this cancer context. To investigate SIX1 function in RMS, we examined the expression of SIX1 in a panel of human RMS cell lines and detected high SIX1 expression in both FN and FP RMS cell lines (Figure 1F). Although SIX1 expression is high in both FP and FN-RMS, we focused our studies on the FN subtype to interrogate its functions outside the context of the PAX3-FOXO1 fusion. Using two FN-RMS cell lines (SMS-CTR and RD) that highly express SIX1, we sought to validate the CRISPR-Cas9 screen findings using an orthogonal method. We thus established SMS-CTR and RD cell lines transduced with shRNAs targeting either no coding sequence in the genome (shScramble) or two distinct SIX1 sequences (SIX1 KD5, SIX1 KD6) (Figure 1G). In both cell lines, we observed that reduced levels of SIX1 were paired with deficits in cell growth and mitotic activity as measured by IncuCyte live-cell growth assays (Figure $1 \mathrm{H}$ ) and the mitotic marker phosphohistone H3 (phH3) staining, respectively (Figure 1I). Together, these data demonstrate that SIX1 is highly expressed and required for the growth of RMS cells in vitro.

\section{six $1 b$ is required for zebrafish RMS tumor growth}

Given the above in vitro observations, we sought to examine the role of SIX1 in an in vivo setting, first using a zebrafish model of ERMS (zRMS) induced by the co-injection of rag2-kRASG12D and rag2-GFP transgenes into the single-cell stage of the zebrafish ${ }^{19}$. This model results in the generation of skeletal muscle tumors with histological features similar to human FN-RMS, and parallels our cell line data, as SMS-CTR and RD cells are both RAS-mutated FN-RMS ${ }^{20,21}$. To examine the expression of the two 
160

161

162

163

164

165

166

167

168

169

170

171

172

173

174

175

176

177

178

179

180

181

182

183

184

zebrafish six1 paralogs, six1a and six1b, in zRMS tumors, we performed quantitative real-time PCR (qRT-PCR) analysis and found that six $1 b$ was significantly upregulated in zRMS tissue compared to agematched normal skeletal muscle (Figure 2A), which was confirmed using RNA in-situ hybridization (ISH) (Figure $2 \mathrm{~B}$ ). To determine whether six $1 b$ was required for RMS tumor growth in vivo, we then combined the zRMS injection model with zebrafish carrying genetic loss-of-function alleles for only six $1 b^{11}$, both because of its more consistent overexpression in zRMS, and because the six1a;six1b double mutant fails to survive to adult stages when zRMS tumors would typically form ${ }^{11,19}$. In contrast, six $1 b$ mutants develop normally and are therefore a suitable model to test the function of reduced six1 levels in RMS in vivo. Consistent with our previous findings, we found no differences in pax3a, myod1 or myogenin expression between wildtype and six $1 b$ mutant sibling embryos from the 5-20+ somite stages (Suppl Fig $2 A-C)^{11}$.

To determine whether six $1 b$ loss is sufficient to alter $k R A S$-mediated zRMS tumorigenesis, we injected rag2-kRASG12D/GFP transgenes ${ }^{19}$ into the progeny of six $1 b^{+/-}$breeding pairs to generate age-matched sibling groups with all possible six1b genotypes. Interestingly, while GFP positivity could be detected in all genotypes, the progression to overt tumors was largely lost with six1b depletion (Figure 2C-E). Following tumor initiation, however, we observed that tumors established in six $1 b^{-/}$zebrafish grew significantly slower over a 120-day time course, as compared to tumors established in wildtype siblings (Figure 2C-D). Reflecting this reduced growth rate, six $1 b^{-/-}$tumors were smaller in size compared to that of wildtype siblings' tumors at their final collection time-point at $120 \mathrm{dpf}$ (Figure 2E). Immunohistochemical staining of tumors demonstrated that while wildtype tumors displayed normal architecture of RMS, six $1 b$ /- tumor cells displayed more elongated morphology with higher cytoplasmic to nuclear ratios, reminiscent of skeletal muscle differentiation (Figure 2F). In alignment with the slow growth rate, staining for phH3 in six $1 b^{-/}(n=3)$ tumors trended toward lowered intensity when compared to prominent phH3-positive staining in wildtype zRMS tumors $(n=4)$. This downward shift did not reach statistical significance $(p=0.081)$ likely due to the to the small number of six $1 b^{-/}$tumors that formed and were evaluable. 
185

186

187

188

189

190

191

192

193

194

195

196

197

198

199

200

201

202

203

204

205

206

207

208

209

210

Nevertheless, the reduction in GFP+ tumor growth in the $\operatorname{six} 1 b^{-/}$zebrafish indicate that six $1 b$ plays a critical role in zRMS tumor progression at least in part via controlling RMS tumor cell proliferation.

\section{SIX1 knockdown inhibits human RMS tumor growth and progression}

The tumors that formed in $\operatorname{six}_{1} b^{-/}$zebrafish displayed an elongated, more spindle-cell morphology, suggesting that RMS cell-state fundamentally differs between RMS cells derived from wildtype and six1b depleted animals. To identify whether similar changes occur in human RMS, we examined the morphology of SMS-CTR and RD cells that were transduced with SIX1 shRNAs. Within approximately five passages after stable SIX1 KD, both RMS cell lines began to exhibit an altered, elongated morphology, distinguishing them from shScramble controls cells (Figure 3A-B).

We next assessed the in vivo outcomes of SIX1 KD in RMS tumor growth. SMS-CTR shScramble and SIX1 KD cells were xenografted subcutaneously in Matrigel into either the left or right flank of immunecompromised NOD/SCID/IL2R $\gamma$ mice and screened weekly for tumor growth. Tumor growth over time, as represented by tumor volume and final tumor weight, was significantly reduced in SIX1 KD tumors compared to shScramble tumors (Figure 3C-E). Histological characterization of the dissected control and SIX1 KD tumors by H\&E revealed clear histological distinctions between shScramble and SIX1 KD tumors whereby all shScramble tumors exhibited high cell density while SIX1 KD tumors were sparsely populated with cells distinguished by elongated nuclear and cytoplasmic morphology (Figure 3F). Notably, upon staining xenografted tumors for phH3, we found that SIX1 KD tumors exhibited significantly less mitotic activity than shScramble tumors (Figure 3G), yet apoptosis, as measured by cleaved caspase 3 (CC3) staining, was unchanged (Suppl Fig 3). These data demonstrate that the profound differences in in vivo tumor growth between shScramble and SIX1 KD RMS tumors can be largely attributed to the lower proliferative capacity of SIX1 KD tumors, and are not due to higher levels of apoptosis.

\section{SIX1 knockdown induces myogenic differentiation in RMS cells}


As described above, loss of SIX1 suppresses in vitro and in vivo RMS growth, and leads to alterations in cell morphology, consistent with morphological changes that occur during myogenic differentiation. Because SIX1 KD induced profound cell elongation and anti-proliferative phenotypes in our RMS cell lines, we asked whether these phenotypes were a consequence of SIX1 directly regulating a proproliferative transcriptional program, or a secondary consequence of another upstream program regulated by SIX1. We hypothesized that similar to its functions in normal skeletal muscle development, SIX1 overexpression in RMS may regulate an early myogenic transcriptional program that supports RMS cell proliferation and self-renewal ${ }^{7,17}$. Therefore, to delineate the transcriptional program coordinated by SIX1 in RMS, we performed RNA-sequencing analysis (RNAseq) on our SMS-CTR shScramble and SIX1 KD cell lines.

The RNAseq analysis revealed a total of 1017 differentially expressed genes (|Fold-change/ $\geq 1.5 \&$ FDR $\leq 0.25$ ) between SMS-CTR shScramble and SIX1 KD cells (Figure 4A). Of note, numerous musclespecification genes such MYOG, MYMK, and MYMX, were marked as significantly upregulated while genes known to regulate cell motility and invasion such as TWIST2 and L1CAM were significantly downregulated ${ }^{22-24}$.To identify dysregulated pathways upon SIX1 KD, we performed gene set enrichment analysis $(\text { GSEA })^{25}$. This analysis revealed an overarching positive enrichment of muscle cell differentiation and contractile muscle gene signatures in SIX1 KD cells (Figure 4B) while chromatin assembly and developmental cell growth signatures were negatively enriched in SIX1 KD cells (Figure 4B, Suppl Fig 4A). Upon closer inspection of gene expression within the MSigDB Myogenesis hallmark pathway, we again observed a clear switch in the expression pattern of canonical myogenic genes from low expression in shScramble cells to higher expression in SIX1 KD cells (Figure 4C).

To validate the changes observed in SIX1 KD cells by RNAseq, we performed qRT-PCR in both SMSCTR and RD cell lines for a subset of differentially expressed myogenic genes identified from our RNAseq 
expressed reduced levels of $P A X 7$ (a TF enriched in muscle progenitors) and expressed higher levels of the myogenic regulatory factors MYOD1, MYOG, and MYF6. In agreement with our RNAseq results, we also observed increased expression of genes associated with myoblast fusion: MYMK and MYMX(Figure 4D $)^{28}$. To further examine whether our SIX1 KD cells underwent myogenic differentiation, we stained SMS-CTR and RD SIX1 KD cells for myosin heavy chain (myHC), a marker of terminal muscle differentiation. In both cell line models, SIX1 KD cells exhibited higher proportions of myHC+ cells (Figure 4E-F) and were more frequently multinucleated than shScramble cells (Figure 4G). These data indicate that SIX1 KD RMS cells are capable of terminally differentiation and forming multinucleated myofibers in contrast to shScramble cells, which maintain their muscle progenitor state.

To determine whether this muscle differentiation phenotype observed with SIX1 loss in human RMS models is conserved in the zRMS model, we additionally stained wildtype and $\operatorname{six}_{1} \mathrm{~b}^{-/} \mathrm{zRMS}$ tumors for Pax7 and myHC. In evaluable wildtype and six $1 b^{--}$tumor sections, we observed a decrease in Pax7 staining in six $1 b^{-/}$tumors compared to wildtype tumors (Suppl Fig 5A), indicative of a shift in differentiation status of the tumors toward a more myotube-like state. In one particular six $1 b^{-/}$tumor, we observed strong myHC staining in the tumor section which contrasted the largely absent myHC staining in all wildtype tumor sections (Suppl Fig 5B). Taken together, these data demonstrate that SIX1 functions to repress a myogenic differentiation program in RMS cells in both human and zebrafish models.

SIX1 globally regulates both stem/oncogenic and myogenic differentiation genes through finetuning of super-enhancer activity

To decipher the mechanism by which SIX1 loss results in transcriptional reprogramming, causing RMS 259 cells to differentiate and stop growing, we performed an initial TF motif analysis using the RCisTarget R 260 package to identify transcriptional regulators with predicted binding within $+/-2.5 \mathrm{~kb}$ of the TSS of the 261 subset of differentially expressed genes. From this analysis, we observed strong enrichment for E-box 262 motifs of which $41 \%$ (350/853) of the genes with expression differences with SIX1 KD were predicted to 
be regulated by the E-box myogenic TFs, MYOD1 and/or MYOG, yet only 4\% (37/853) of these genes were predicted to be directly regulated by SIX1 (SuppI Fig 6). Thus, we hypothesized that SIX1 loss leads to differentiation of RMS cells via reprogramming of myogenic TFs.

To determine how loss of the SIX1 TF activates a myogenic differentiation program, we performed chromatin immunoprecipitation followed by sequencing (ChIPseq) using a polyclonal antibody made against SIX1. We also performed ChIPseq against the master regulator of the myogenic lineage, MYOD1, and the active enhancer/chromatin histone mark H3-lysine-27 acetylation (H3K27ac) in SMS-CTR shScramble and SIX1 KD cell lines. Reflecting levels of shRNA-mediated SIX1 KD, we observed reduced genome-wide binding of SIX1 in both SIX1 KD lines compared to shScramble cells (Figure 5A) and sites of reduced SIX1 binding were highly enriched for SIX1/2 consensus motifs (Figure 5B). We further annotated genetic loci exhibiting 1.5-fold reduced SIX1 binding in both SIX1 KD lines compared to the shScramble control and found that SIX1 binding was reduced at gene loci involved in stem cell differentiation, Ras signaling, and cytoskeletal organization (Figure 5C). Accompanying sites of reduced SIX1 binding, we additionally observed decreases in MYOD1 and H3K27ac signal (Figure 5D, Suppl Fig 7). These data suggest that SIX1 predominantly plays a transcriptional activating role in FN-RMS and that SIX1 KD leads to a reduction in transcriptional output at stem-related and Ras-driven genes.

Given the changes in H3K27ac deposition and MYOD1 binding upon SIX1 KD, we hypothesized that SIX1 likely regulates large-scale transcriptional programs through mechanisms beyond direct transcriptional induction of cis genes. To determine how SIX1 KD affects global transcriptional output, we annotated H3K27ac signal distribution over promoters (+/- 2.5kb from TSS), gene bodies, and distal enhancers. In SIX1 KD cells, H3K27ac distribution increased at promoter regions (+/-2.5kb of TSS) and was reduced along gene bodies and moderately reduced at distal intergenic regions/enhancers (Figure $2875 \mathrm{E})$, showing a potential function of SIX1 in regulating enhancer activity in addition to promoter-based 288 transcription. 
To examine whether SIX1 levels influences enhancer activity, we compared enhancers and superenhancers (SEs) via ranked H3K27ac signal between shScramble and differentiated SIX1 KD cells. Overall, 4.14\%, 5.24\%, and 7.37\% of total H3K27ac peaks in shScramble (1470), SIX1 KD5 (1452), and SIX1 KD6 (1322) cells respectively corresponded to super-enhancers, which are characterized by longranging (over 12.5kb) clusters of strong H3K27ac deposition ${ }^{29,30}$. Of note, we found that many oncogenic and myogenic genes marked as differentially expressed upon SIX1 KD in our RNAseq dataset were associated with SEs. For example, in SIX1 KD cells, we observed a downward shift in ranked H3K27ac signal at the SE associated with the Notch effector and muscle stem cell enriched gene $H E Y L^{31}$, and an upward shift of H3K27ac signal at the SE associated with the contractile muscle genes TNNT2 and TNNI1, denoted as the TNNT2 SE by the Rank Ordering of Super Enhancers (ROSE) algorithm (Figure 5F). We further annotated shScramble and SIX1 KD SEs by closest neighboring genes and discovered that although SEs occurred at myogenic-associated genes in both conditions, myogenic SEs in both SIX1 KD cell lines were associated with structural and contractile functions of skeletal muscle whereas those in the shScramble cell line were associated with less differentiated skeletal muscle pathways (Figure 5G). Side-by-side comparison of H3K27ac and SIX1 binding tracks at the example HEYL and TNNT2/TNNI1 SEs not only reflects the shifts in SE activity seen in Figure 5F, but also demonstrates that SIX1 occupancy follows the pattern and trend of H3K27ac deposition (Figure 5H). Intriguingly, despite having global reduction in SIX1 binding and H3K27ac signal overall (Figure 5A, Suppl Fig 7), SIX1 binding and H3K27ac deposition at the TNNT2/TNNI1 SE increased in the SIX1 KD5 line and remained relatively unchanged in the SIX1 KD6 line in comparison to that of the shScramble line. In contrast, SIX1 and H3K27ac signal at the HEYL SE were consistently reduced in both SIX1 KD lines, which contributed to the downward shift in HEYL SE rank in both SIX1 KD lines and reduced HEYL expression (Figure 5F\&H).

312 When examining the effects at a more global level, we observed similar reductions of H3K27ac signal at 313 SEs associated with stem-related genes and relatively unchanged H3K27ac signal at SEs associated 314 with muscle differentiation (Suppl Fig 7B). These data suggest that a loss of SE activity at stem genes 
may be the driving force of differentiation during SIX1 KD. As the SIX1 antibody used in ChIP has been shown to cross-react with other highly related SIX family members ${ }^{32}$, we reason that the lack of a decreased SIX1 binding at the TNNT2/TNNI1 could be due to differences in SIX1 affinity to the myogenic loci during the differentiation state, or to the presence of a compensatory SIX member which could be recognized by the ChIP antibody. Nonetheless, these findings in the context of SIX1 loss of function, demonstrate a role for SIX1 in fine-tuning the activity of myogenic SEs that govern myogenic commitment as well as differentiation into contractile fibers.

\section{SIX1 loss alters MYOD1 occupancy at muscle differentiation and stem/oncogenic loci}

By regulating SE activity, we reasoned that accessibility of myogenic TFs at oncogenic and myogenic loci could be affected by SIX1 KD. We observed that loss of SIX1 resulted in a change in MYOD1 distribution from distal intergenic/enhancer to promoter regions (Figure 6A). This coincides with the change in H3K27ac, indicating that SIX1 loss alters transcriptional dynamics, resulting in enhanced promoter-based and reduced enhancer-based transcription. Nearest gene annotation of MYOD1 peaks in the shScramble cell line and overlapping MYOD1 peaks in the SIX1 KD cell lines demonstrated that MYOD1 remains bound to myogenic loci in both shScramble and SIX1 KD genomes (Figure 6B). However, in the setting of reduced SIX1, we observed that MYOD1 sites occupied loci involved in positive regulation of muscle differentiation which did not appear in the top 10 pathways of shScramble MYOD1 peak (Figure 6B). Examples of the shift in MYOD1 binding are shown at the MYMK and NOTCH3 loci (Figure 6C). Particularly at the MYMK locus, which is a key gene involved in myoblast fusion and formation of multinucleated myotubes ${ }^{28,33}$, MYOD1 binding occurs at the gene promoter, and increases upon SIX1 KD (Figure 6C), which is consistent with its increased expression in SIX1 KD cells (Figure 4A\&D, 6C). At the NOTCH3 loci, MYOD1 binding occurs 22kb downstream of SIX1 within the NOTCH3 promoter region and dramatically decreases upon SIX1 KD without a significant reduction in SIX1 binding at the upstream enhancer site and is coupled with downregulated mRNA expression (Figure 6C). In both these cases, we observed that changes in MYOD1 occupancy, rather than SIX1, aligned with H3K27ac 
marks. To validate the shift in MYOD1 occupancy at differentiation and progenitor-related genes in both SMS-CTR and RD cells, we performed MYOD1 Cleavage Under Targets and Release Under Nuclease (CUT\&RUN) followed by qPCR (C\&R qPCR), which is an orthogonal method to ChIP to detect target protein binding on DNA and requires far less cells than traditional ChIP methods ${ }^{34}$. We found that in differentiated SIX1 KD SMS-CTR cells as well as RD cells, MYOD1 was more abundantly bound at loci associated with differentiation genes, as opposed to myoblast or oncogenic genes (Figure 6D). These results reflect similar observations of MYOD1 genomic occupancy shifting as a consequence of myoblast formation or RMS induction toward differentiation ${ }^{3,13,14,35,36}$. Thus, our data demonstrate that SIX1 regulates a large-scale proliferative and less differentiated cell-identity program in RMS by maintaining MYOD1 binding at SEs resulting in a loss of promoter-driven myogenic gene transcription. Thus, SIX1 loss leads to an altered myogenic TF DNA binding landscape to one that is more permissive to the expression of contractile muscle genes over the expression of stem-related genes regulated by SEs.

\section{SIX1 expression is inversely correlated with a Myotube gene signature in RMS patients}

The profound myogenic transcriptional program induced upon SIX1 inhibition suggests that the overexpression of SIX1 may serve as an upstream orchestrator of the aberrant muscle differentiation observed in RMS, as it does in normal muscle development ${ }^{8}$. To test this, we examined whether SIX1 expression in RMS patient samples correlates with an early myogenic transcriptional landscape. Using a recently published human pluripotent stem cell (hPSC) dataset ${ }^{37}$ aimed at defining the transcriptional landscape at multiple stages of human myogenic differentiation, we derived a myogenic differentiation signature from PAX7+ skeletal muscle progenitors and their final cell states as multinucleated myotubes. With this hPSC data to serve as case-controls for differentiated muscle and muscle progenitors, respectively, we applied a signature scoring method ( $S$-score) previously described by Hsiao and colleagues $^{38}$ to quantitatively score test data, RMS patient RNAseq samples, on their concordance to the gene expression signatures derived from empirical myotube-progenitor data (Figure 7A). To test the 
scoring could segregate PAX7+ progenitors, MYOG+ myoblasts, and differentiated myotubes in a stepwise manner whereby the MYOG+ cells displayed an intermediate $S$-score between muscle progenitors and myotubes (Figure 7B). Furthermore, we calculated an S-score for our SIX1 KD RNAseq samples based on the myotube signature and were able to distinguish shScramble from SIX1 KD RMS cells based on this scoring method. SIX1 KD cells demonstrated greater alignment with the myotube signature, consistent with the results of other enrichment scoring methods used previously in Figure 4 (Figure 7C). Importantly, using this quantitative scoring technique, we are able to assess what stage of the myogenic differentiation cascade our RMS cells lie.

We next assessed how SIX1 expression correlates with myotube S-scores in RMS patient samples. In the St Jude iRDb cohort, we found a modest and statistically significant inverse correlation between SIX1 expression and myotube $S$-Scores (Figure 8D, Spearman correlation: $R=-0.36, p=0.0012$ ). We additionally applied the same signature scoring algorithm to generate a SIX1 KD signature using our

SIX1 KD RNAseq dataset as case (KD)-controls (shScramble) and S-scored both St Jude and strong positive correlations (St Jude: $\mathrm{R}=0.57, p<0.001$, GSE108022: $\mathrm{R}=0.61, p<0.001$ ) between the two signatures in the RMS patients, indicating that loss of SIX1 expression in RMS cells induces a transcriptional program highly similar to that which is observed by a myoblast transitioning to the myotube fate (Figure 7E).

Given the concordance of the SIX1 KD signature with the myotube signature, we next sought to examine whether these two signatures could be used to distinguish advanced RMS disease from primary disease. Of the 71 patient samples with complete RNAseq data available from the St Jude iRDb cohort, three of these patients had RNA-sequencing performed at multiple stages of the patient's disease progression. 391 Filtering down our analysis to these three patients, we examined whether disease recurrence was 392 associated with changes in myogenic differentiation state. By myotube and SIX1 KD S-scoring, we 
observed that patient tumor expression profiles at diagnoses and disease recurrence states were distinguishable by differentiation and SIX1 KD scores, whereby relapsed tumors exhibited lower SIX1KD and myotube $S$-scores than their tumor at diagnosis (Figure 7F). Of note, we observed that the two relapsed tumor samples from patient B012 had lower Myotube and SIX1 KD S-scores compared to the tumor at diagnosis (Figure 7F). These data underscore our findings that the transcriptional program controlled by SIX1 in RMS is intimately linked to myogenic differentiation status, which is a driving force of RMS tumor progression.

\section{Discussion}

402 Repression of myogenic differentiation programs is a known, critical attribute of RMS whereby dysfunctional MYOD1 and MYOG activity is thought to drive the disease ${ }^{3,39-42}$. An unresolved question that persists in the field of RMS is why RMS tumors express the myogenic TFs, MYOD1 and MYOG, yet fail to progress past the apparent myoblast progenitor state $2,43,44$. While it is known that MYOD1 and MYOG have distinct subprograms that can drive either self-renewal or skeletal muscle differentiation, the departure of these MRFs from their canonical abilities to execute the complete sequence of skeletal muscle development in RMS invokes other factors that may repress the ability of MYOD1 to act on its differentiation programs. Therefore, the identification of other regulatory proteins that alter the contextspecific functions of MYOD1 has become a core area of RMS studies ${ }^{3-5}$. Here, we report that the SIX1 homeobox TF acts as an upstream transcriptional regulator maintaining the arrest of RMS cells in a selfrenewing muscle progenitor state. In the developmental context, the SIX1 homeobox gene is highly expressed in early muscle development and is responsible for the direct activation of early MRF expression, but its expression becomes downregulated as the muscle reaches its final stages of 415 differentiation ${ }^{45,46}$. Using zebrafish and human cell line FN RMS models, we demonstrate that genetic 416 inhibition of $S I X 1 /$ six $1 b$ can trigger the activation of a muscle differentiation gene program in RMS cells, 417 thus halting their growth and spread. These data are supported by preceding reports that show 
downregulation of SIX1 occurs during the final stages of muscle differentiation and embryonic myogenesis ${ }^{11,37,45,46}$, and further supports the hypothesis that aberrant SIX1 expression in RMS may be in part responsible for the MRF dysfunction occurring in RMS.

In the majority of studies implicating the role of $S I X 1$ in cancer progression, SIX1 ostensibly acts as a TF that induces the expression of downstream tumor-promoting genes. Notably, in two previous reports, the pro-metastatic functions of Six1 in Rhabdomyosarcoma (RMS) were reported to be channeled through one of Six1's transcriptional targets, Ezrin, a cytoskeletal protein ${ }^{47,48}$, which was proposed to alter migration and invasion and thus contribute to RMS progression. In this study, we show for the first time that SIX1 promotes tumor growth/progression largely via alteration of global transcriptional programs of muscle cell-identity. Thus, while direct targets such as Ezrin likely contribute to its aggressive functions in RMS, the major function of SIX1 in RMS progression appears to be through changing cell fate by regulating transcriptional programs upstream of myogenic TFs. In normal development, Six 1 loss in muscle precursor cells leads to reduced MRF expression and concomitant defects in skeletal muscle formation $7,8,11,16,17,49,50$. In the context of FN-RMS, we observe that SIX1 KD is associated with loss of progenitor gene expression but a gain of muscle differentiation gene expression, raising the question of how SIX1 activates a differentiation program while it is itself suppressed. By ChIPseq, we observe that genome wide SIX1 binding closely overlaps with H3K27ac marks at promoters and SE regulatory elements. SIX1 KD leads to decreases in SIX1 binding at cytoskeletal, cell division, and stem-related loci, which aligns with previously characterized roles of SIX147,48,51-54. On a global scale, SIX1 binding is enriched at SEs, enhancers, and promoters associated with cell division, cell-identity, and muscle specification. Upon SIX1 KD, SE activity as approximated by H3K27ac signal is diverted from progenitor/stem-related SEs to SEs associated with that of forming contractile muscle and other structural components of skeletal muscle differentiation, which manifest as the multinucleated and elongated morphology of SIX1 KD cells. In addition to these direct forms of transcriptional regulation either at target loci or at distal regulatory elements, we found that SIX1 can indirectly influence the DNA binding activity 
444

of MYOD1 and possibly other myogenic TFs by modifying the landscape of active chromatin and consequently TF binding accessibility at differentiation loci.

Pluripotency and cell type determination are controlled by the occupancy of master TFs and cell-type specific TFs at enhancer regions governing cell fate decisions $\mathrm{s}^{29,55}$. Within the repertoire of muscle-lineage enhancers, several TFs, which based on our studies include $S I X 1$, have come to light as master TFs that initialize the myogenic lineage by sitting poised at myoblast enhancer elements and then become overactive in the context of RMS ${ }^{35,36,39,42}$. Notably, these factors include the developmental TFs SNA11/2 and TWIST2, which similar to SIX1 are found at stem and myogenic enhancer elements in RMS and are drivers of EMT, cell migration, and tissue repair ${ }^{13,35,36}$. Our focused study of $S I X 1$ compounds on growing evidence that the composition of TFs at muscle-specific enhancers controls the differentiation state of RMS cells, which raises multiple outstanding questions. First, this raises the question of what factors cause SIX1 to become overexpressed in FN-RMS tumors, particularly given the absence of SIX1 amplification or any common perturbation of the locus. Whereas $S I X 1$ has been identified as target downstream of the PAX3-FOXO1 fusion, the mechanism leading to SIX1 overexpression in FN-RMS is less understood ${ }^{56}$. Second, our findings raise the question of how diverse driver mutations associated with FN-RMS impinge on similar myogenic epigenetic/transcriptional programs in similar fashion to the PAX3-FOXO1 fusion protein in FP-RMS ${ }^{57-59}$. Notably, genome-wide PAX3-FOXO1 fusion binding establishes SEs at myogenic genes and recruits the co-activator proteins p300, BRD4, and Mediator ${ }^{59}$, and similar functions may apply to TFs like SIX1 in FN-RMS . Finally, the collection of these studies raises the question of whether RMS cells can be irreversibly reprogrammed to follow the proper cascade of myogenic differentiation through targeting master TF activity. Although there are still many barriers facing the viability of TFs as pharmacological targets, dissection of mechanisms that modulate specific TF activities can potentially reveal druggable nodes that control cell-type specific transcriptional programs. For example, the requirement of an EYA phosphatase co-factor interaction with SIX1 to strongly activate downstream target transcription represents one targetable node to SIX1 activity that our group is actively 
470 interrogating ${ }^{60-63}$. Thus, it will be of future interest to determine whether the EYA phosphatase plays a 471 similar role together with SIX1 in trapping RMS cells in a progenitor-like state.

472

473 In summary, our studies demonstrate that the SIX1 TF prevents FN-RMS from undergoing the cascade 474 of myogenic gene expression leading to differentiation via the regulation of transcriptional output at stem 475 versus myogenic genes. We show that FN-RMS differentiates into non-proliferative myotube-like cells 476 following SIX1 inhibition, and that the differentiation program is achieved by a shift in MYOD1 binding 477 and enhanced transcriptional activity from genetic loci that foster cell growth to loci that specify and drive 478 the myogenic lineage. Altogether, these findings define an epigenetic function of SIX1 in balancing the 479 growth and differentiation properties intrinsic to the myogenic lineage and ultimately demonstrate SIX1 480 as suitable therapeutic target in RMS. 


\section{Main Figure Legends}

492

493

494

495

496

497

498

499

500

501

502

503

504

505

506

507

508

509

510

511

512

513

514

515

Figure 1. SIX1 is overexpressed and predicted to be an essential gene in Rhabdomyosarcoma (A) Fragments per kilobase million (FPKM) expression of SIX1 in the St. Jude Pediatric Cancer Genome Project cohort (Grey = three normal skeletal muscle controls; FQ21 = fetal quadricep muscle). (B) IHC staining counterstained with hematoxylin and DAB intensity scoring of an RMS tumor array with normal skeletal muscle controls. (C) Frequency distribution of IHC scores across RMS and skeletal muscle tissue cores and frequency distribution of tissue cores with IHC scores $\geq 2$. (D) SIX1 transcripts per kilobase million (TPM) expression against SIX1 gene effect score in 1775 cell lines in the Cancer Dependency map CRISPR-Cas9 large-scale KO screen (RMS cell lines in blue). (E) Volcano plot of gene dependency scores for MYOD1 (blue) and SIX1 (red) in RMS cell lines versus all other cell lines of different tissue types. Statistical analysis of gene dependencies between RMS and all other cell types were performed using a two-class Kolmogorov-Smirnov test. (F) Western blot of SIX1 protein levels across a panel of FN and FP RMS human cell lines (G) shRNA-mediated knockdowns of SIX1 in RD and SMS-CTR cell lines. $(H)$ IncuCyte live-cell imaging growth assays of SMS-CTR and RD shScramble and SIX1 KD cells over a 96-hrs. Cells were plated in triplicate and relative cell growth was measured by normalizing cell confluency at each time point relative to initial timepoint confluency. Data represent mean \pm SEM and statistical differences between shScramble and SIX1 KD5 or SIX1 KD6 was measured by fitting data to a longitudinal mixed effects model. (I) Mitotic activity of SMS-CTR and RD shScramble and SIX1 KD cells measured by phH3 staining. Cells were counterstained with DAPI. Data represent mean \pm SEM of at least 3 independent experiments.

\section{Figure 2. six1b is required for zebrafish RMS tumor growth}

(A) qRT-PCR expression of zebrafish six1 paralogs six1a and six $1 b$ in dissected GFP + zRMS tumor tissue compared to age-matched normal skeletal muscle ( $n=4$ normal muscle samples, $n=6$ zRMS tumor samples). (B) Representative images of six1b transcripts as visualized by H\&E and in situ 
516 hybridization signal (purple puncta; $n=5$ fish per group) (C) Representative images of tumor

517 progression (outlined in green) over 28 days from 57-85 days post fertilization (dpf) between wildtype 518 and $\operatorname{six} 1 b^{-/}$tumor-burdened individuals. Yellow outline represents autofluorescence from stomach and 519 yolk. (D) Quantification of tumor area by GFP+ tumor area in each individual fish over time. Tumor 520 growth per individual is represented as individual tracks and composite growth of wildtype and six $1 b^{-/}$ 521 tumors was fitted to a non-linear logistical growth model and represented by dotted lines. A longitudinal 522 mixed effect model was used to measure statistical differences between conditions over repeated 523 measures. (E) Tumor area growth over time normalized to standard length of fish at $120 \mathrm{dpf}$ or at prior 524 time point due to moribundity. (F) Representative staining and quantification of H\&E and phospho525 histone H3 IHC (brown) in sectioned zRMS tumors. Dots in graph represent \%phH3 staining per tumor section; phH3 staining quantified over 2 sections per tumor ( $n=4$ wt tumors, $n=3$ six $1 b^{-/-}$tumors). Statistical differences were calculated using a Welch's $t$-test.

\section{Figure 3. SIX1 knockdown inhibits human RMS tumor growth and progression}

(A) Brightfield images depicting elongated cell morphology of SIX1 KD SMS-CTR and RD cells along with (B) quantification of cell lengths. (C) Tumor volumes, measured by caliper, over a 12-week time period of shScramble and SIX1 KD SMS-CTR cells that were engrafted bilaterally into the flanks of NOD/SCID $\gamma$ (NSG) mice. Data represent mean \pm SEM and were fitted to a longitudinal mixed effects model for statistical analysis of shScramble and SIX1 KD samples (D) Representative images of dissected shScramble or SIX1 KD xenografted tumors at 12 weeks. (E) Final tumor weights in grams at the end of the 12-week study. ( $n=10$ mice total; 10 mice received shScramble cells in one flank, and SIX1 KD cells in opposite flank. 5 mice received a SIX1 KD5 flank injection, and 5 mice received a SIX1 KD6 flank injection). (F) Representative H\&E histology of dissected shScramble and SIX1 KD xenografted tumors. (G) Representative phH3 immunostaining (brown) of dissected shScramble and staining quantified over 2 sections per tumor. 
Figure 4. SIX1 knockdown induces myogenic differentiation in RMS cells

(A) Volcano plot of $\log _{2}$ fold-change (FC) gene expression (SIX1 KD over shScramble) and adjusted $p$ value after edgeR-based differential expression analysis from the SMS-CTR RNAseq experiment. Red dots denote genes significantly upregulated ( $F C \geq 1.5 \&$ adj $p$-value $\leq 0.25)$ and blue dots denote genes significantly downregulated (FC $\leq-1.5 \&$ adj p-value $\leq 0.25)$ upon SIX1 KD. (B) Gene set enrichment analysis plots of ranked log2FC expression (SIX1 KD over shScramble) show positive enrichment for curated muscle cell differentiation and skeletal muscle contraction gene signatures and negative enrichment for chromatin assembly gene signatures. (C) Heatmap plotting expression of the MSigDB myogenesis gene set across shScramble and SIX1 KD samples. Scale bar represents z-scoreconverted log2CPM values. (D) Validation of differential mRNA expression of genes involved in muscle differentiation in SMS-CTR and RD cell lines with SIX1 KD by qRT-PCR. Barplot data represent mean \pm SEM expression values across $n \geq 5$ independently collected biological samples. (E) Positive MyHC (MF-20, red) immunostaining and DAPI counterstain (blue) in SIX1 KD RMS cells compared to shScramble RMS cells. (F) Quantification of myHC staining over total nuclei per field of view (each dot represents \%myHC+ cells over one technical replicate from at least 3 independent experiments) and (G) fusion indices of SMS-CTR and RD control and SIX1 KD cells.

\section{Figure 5. SIX1 globally regulates both stem/oncogenic and myogenic differentiation genes}

\section{through fine-tuning of super-enhancer activity}

(A) Heatmaps of genome-wide SIX1 ChIPseq signal in SMS-CTR shScramble, SIX1 KD5, and SIX1

KD6 cells. Heatmaps were generated using deepTools and centered at shScramble SIX1 peaks. (B)

Motif analysis on peak coordinates exhibiting reduced 1.5-fold SIX1 binding in both SIX1 KD5 and SIX1 KD6 SMS-CTR SIX1 ChIPseq datasets. Top 4 enriched motifs shown. (C) Pathway enrichment plots of annotated sites of SIX1 loss in both SIX1 KD5 and KD6 cell lines. Enrichment plots were generated using ChIPseeker followed by ClusterProfiler R packages with gene set sizes restricted to 100 to 250 genes and a q-value cut-off of 0.05. (D) ChIPseq average profiles of MYOD1, and H3K27ac signal over 
568

569

570

571

572

573

574

575

576

577

578

579

580

581

582

583

584

585

586

587

588

589

590

591

592

593

SIX1 binding sites that exhibited reduced binding in SIX1 KD cells compared to shScramble cells. Average profiles were centered around reduced SIX1 peaks and show co-occurrence of SIX1 and MYOD1 binding as well as H3K27ac deposition in SMS-CTR cells. (E) Peak distribution of H3K27ac signals in SMS-CTR shScramble, SIX1 KD5, and SIX1 KD6 cells across promoters (+/-2.5kb from annotated TSS), 5/3' UTR, gene body, and distal intergenic/enhancer regions. (F) ROSE analysis performed on shScramble and SIX1 KD H3K27ac ChIP peaks depicts the shift in HEYL (down) and TNNT2/TNNI1 (up) SE rank between shScramble and differentiated SIX1 KD cells. Although not defined as an SE (top right quadrant of hockey stick plot), the LGR5 enhancer also shifts downward in SIX1 KD cells and is a gene associated with self-renewal and stem properties. (G) Pathway enrichment of genes associated with SEs identified in shScramble and the union of SEs identified in SIX1 KD5 and SIX1 KD6 (SIX1 KD) cells using gene set sizes restricted to 100 to 250 genes and a q-value cut-off of 0.05. (H) H3K27ac and SIX1 ChIP signal over the HEYL and TNNT2/TNNI1 SEs depict changes in SIX1 binding abundancy at stem cell (HEYL) and muscle differentiation (TNNT2/TNNI1) loci during SIX1 KD-induced differentiation and respective levels of HEYL and TNNT2/TNNI1 expression as observed in the RNAseq data. ChIPseq tracks were generated using the Washington University Epigenome Browser.

\section{Figure 6. SIX1 loss alters MYOD1 occupancy at muscle differentiation and stem/oncogenic loci} (A) Peak distribution of the MYOD1 TF in SMS-CTR shScramble, SIX1 KD5, and SIX1 KD6 cells across promoters (+/-2.5kb from annotated TSSs), 5/3' UTR, gene body, and distal intergenic/enhancer regions. (B) Pathway enrichment of annotated MYOD1 peaks that were called in shScramble and the union of MYOD1 peaks called in SIX1 KD5 and SIX1 KD6 cells (SIX1 KD). (C) H3K27ac, MYOD1, and SIX1 ChIPseq tracks over the MYMK and NOTCH3 loci depict changes in MYOD1 binding that occur downstream of SIX1 loss and correlate with upregulation of MYMK and downregulation of NOTCH3 expression. ChIPseq tracks were generated using the Washington University Epigenome Browser. (D) CUT\&RUN qPCR validation of changes in MYOD1 binding at stem/oncogenic (HEYL, NOTCH3, 
EGFR), and myogenic differentiation genes (MYMK, MYLK2, TNNT2) that occur in SMS-CTR and RD SIX1 KD5 cells. Statistical differences were measured using a two-way ANOVA test followed by a posthoc Sidak's multiple comparisons test.

\title{
Figure 7. SIX1 expression in RMS is inversely correlated with a myotube gene signature
}

(A) Overview of S-scoring methodology whereby gene expression in the case-control (hPSC differentiated myotubes and Pax7+ progenitors, respectively) group is used to generate a weighted gene signature to score test sample transcriptomes on a continuous scale. (B) Myotube $S$-scores for samples used in training set plotted as proof-of-concept that the Myotube $S$-score can quantify myogenic differentiation status. Statistical differences measured by two-side Student's $t$-tests. (C) Myotube $S$ scoring methodology applied to SIX1 KD RNAseq dataset demonstrates that SIX1 KD cells are more advanced in myogenic lineage than shScramble cells. Statistical differences measured by two-side Student's t-tests. (D) Scatter plot of Myotube S-score plotted against SIX1 z-score-converted expression and Spearman rank correlation coefficient depict a moderate inverse correlation between differentiation status and SIX1 expression in St Jude iRDb RNAseq patient samples $(n=71)$. (E) Scatter plot of SIX1

KD S-scores derived from SIX1 KD RNAseq data against Myotube S-score shows a strong positive correlation between the SIX1 KD and myotube gene signatures in the St Jude iRDb expression dataset. (F) Myotube and SIX1 KD S-scores of three patient tumors (SJRHB011 = "B011", SJRHB012 = "B012", SJRHB026 = "B026") collected and sequenced at multiple disease stages.

\section{STAR Methods}

\author{
Key Resources
}

REAGENT or RESOURCE

SOURCE

IDENTIFIER 


\begin{tabular}{|c|c|c|}
\hline \multicolumn{3}{|l|}{ Antibodies } \\
\hline Mouse monoclonal anti-SIX1 $(1229,992)$ & In-house purified antibody & $\mathrm{N} / \mathrm{A}$ \\
\hline Rabbit polyclonal anti-SIX1/2 & Atlas Antibodies & HPA0011893; AB_1079991 \\
\hline Rabbit polyclonal anti-H3K27ac & Abcam & ab4729; AB_2118291 \\
\hline Rabbit monoclonal anti-MYOD1 & Abcam & ab133627; AB_2890928 \\
\hline Mouse monoclonal anti-myosin heavy chain & DSHB & MF-20;AB_2147781 \\
\hline Mouse monoclonal anti-PAX7 & DSHB & PAX7; AB_528428 \\
\hline Rabbit polyclonal anti-phosphohistone H3 (pSer10) & Sigma-Aldrich & H0412; AB_477043 \\
\hline Rabbit polyclonal anti-cleaved caspase 3 & Cell Signaling Technology & $9661 ; A B \_2341188$ \\
\hline Normal Rabbit lgG & Cell Signaling Technology & 2729; $A B \_1031062$ \\
\hline Mouse $\beta$-TUBULIN & Sigma-Aldrich & T4026; AB_477577 \\
\hline Mouse $\beta$-ACTIN & Sigma-Aldrich & A5316; AB_476743 \\
\hline Mouse $\beta$-ACTIN-HRP & Abcam & ab49900; $\overline{A B} \_867494$ \\
\hline \multicolumn{3}{|l|}{ Bacterial and virus strains } \\
\hline Subcloning Efficiency DH5 $\alpha$ competent cells & ThermoFisher & 18265017 \\
\hline \multicolumn{3}{|l|}{ Biological samples } \\
\hline $\begin{array}{l}\text { Rhabdomyosarcoma with striated muscle tumor } \\
\text { array }\end{array}$ & Biomax & SO2082b \\
\hline \multicolumn{3}{|l|}{ Chemicals, peptides, and recombinant proteins } \\
\hline Polybrene & Millipore & TR-1003 \\
\hline Phenol-red solution & Sigma & P0290 \\
\hline Puromycin Dihydrochloride & Research Products Int. & P33020 \\
\hline pAG-MNase & EpiCypher & $15-1116$ \\
\hline Fugene Transfection Reagent & Promega & E2311 \\
\hline Tricaine (MS-222) & Sigma Aldrich & A5040 \\
\hline ECL Western blot substrate & Pierce & 32106 \\
\hline Digitonin & Millipore Sigma & $30-041$ \\
\hline Spermidine & Sigma Aldrich & S0266 \\
\hline \multicolumn{3}{|l|}{ Critical commercial assays } \\
\hline MycoAlert detection kit & Lonza & LT07-418 \\
\hline Direct-zol RNA prep kit & Zymo Research & R2052 \\
\hline iScript reverse transcription kit & Bio-Rad & 1708841 \\
\hline SsoFast EvaGreen supermix & Bio-Rad & 1725205 \\
\hline Verso cDNA synthesis kit & ThermoFisher & $A B-1453 A$ \\
\hline Taqman gene expression master mix & Applied Biosystems & 4369542 \\
\hline Nuclei EZ prep kit & Sigma Aldrich & NUC101 \\
\hline Dynabeads Antibody Coupling kit & ThermoFisher & $14311 \mathrm{D}$ \\
\hline Concavalin A beads & EpiCypher & $21-1401$ \\
\hline Universal Plus mRNA-Seq library prep kit & Nugen & 0508 \\
\hline KAPA HyperPrep ChIP library kit & Roche & KK8502 \\
\hline NEBNext II Ultra library prep kit & NEB & E7645, E7600S \\
\hline DNA Clean and Concentrator kit & Zymo Research & D4033 \\
\hline \multicolumn{3}{|l|}{ Deposited data } \\
\hline SIX1 KD RNAseq & This paper & $\mathrm{N} / \mathrm{A}$ \\
\hline SIX1 KD ChIPseq & This paper & N/A \\
\hline Pan-Sarcoma and normal tissue expression & $\begin{array}{l}\text { Downloaded from } \\
\text { Oncogenomics database }\end{array}$ & $\begin{array}{l}\text { https://fsabcl- } \\
\text { pob01p.ncifcrf.gov/cgi-bin/JK }\end{array}$ \\
\hline
\end{tabular}




\begin{tabular}{|c|c|c|}
\hline Pediatric Sarcoma expression & $\begin{array}{l}\text { Downloaded from St. Jude } \\
\text { PeCAN portal }\end{array}$ & $\begin{array}{l}\text { https://pecan.stjude.cloud/pro } \\
\text { teinpaint/study/pan-target }\end{array}$ \\
\hline Rhabdomyosarcoma patient RNAseq & $\begin{array}{l}\text { Downloaded from St. Jude } \\
\text { Integrated RMS Database }\end{array}$ & $\begin{array}{l}\text { https://pecan.stjude.cloud/pro } \\
\text { teinpaint/study/RHB2018 }\end{array}$ \\
\hline hPSC muscle differentiation RNAseq & 37 & GSE129505 \\
\hline \multicolumn{3}{|l|}{ Experimental models: cell lines } \\
\hline HEK293T & ATCC & CVCL_0063 \\
\hline Human: $\mathrm{RH} 30$ & Mark Hatley ${ }^{64}$ & CVCL_0041 \\
\hline Human: RH3 (RH28) & Mark Hatley & CVCL_L415 \\
\hline Human: $\mathrm{RH} 4$ & Mark Hatley & CVCL_5916 \\
\hline Human: RD & Mark Hatley & CVCL_1649 \\
\hline Human: RH36 & Mark Hatley & CVCL_M599 \\
\hline Human: $\mathrm{RH} 2$ & Mark Hatley & CVCL_A460 \\
\hline Human: SMS-CTR & Mark Hatley & CVCL_A770 \\
\hline Human: SMS-CTR stable shScramble & This paper & $\mathrm{N} / \mathrm{A}$ \\
\hline Human: SMS-CTR stable shSIX1 KD5 & This paper & $\mathrm{N} / \mathrm{A}$ \\
\hline Human: SMS-CTR stable shSIX1 KD6 & This paper & $\mathrm{N} / \mathrm{A}$ \\
\hline Human: RD stable shScramble & This paper & $\mathrm{N} / \mathrm{A}$ \\
\hline Human: RD stable shSIX1 KD5 & This paper & $\mathrm{N} / \mathrm{A}$ \\
\hline Human: RD stable shSIX1 KD6 & This paper & $\mathrm{N} / \mathrm{A}$ \\
\hline \multicolumn{3}{|l|}{ Experimental models: organisms/strains } \\
\hline Zebrafish: AB & ZIRC & ZL1 \\
\hline Zebrafish: six $1 b^{o z 1}$ & Sharon Amacher ${ }^{11}$ & $\mathrm{~N} / \mathrm{A}$ \\
\hline Mouse: NOD/SCID $\gamma$ & CU AMC Breeding Core & $\mathrm{N} / \mathrm{A}$ \\
\hline \multicolumn{3}{|l|}{ Oligonucleotides } \\
\hline $\begin{array}{l}\text { For SYBR cDNA primer sequences, see } \\
\text { Supplemental Table } 1.1\end{array}$ & This paper & $\mathrm{N} / \mathrm{A}$ \\
\hline $\begin{array}{l}\text { For SYBR CUT\&RUN primer sequences, see } \\
\text { Supplemental Table } 1.2\end{array}$ & This paper & $\mathrm{N} / \mathrm{A}$ \\
\hline $\begin{array}{l}\text { For Taqman Primer/Probe sequences, see } \\
\text { Supplemental Table } 1.3\end{array}$ & This paper & $\mathrm{N} / \mathrm{A}$ \\
\hline \multicolumn{3}{|l|}{ Recombinant DNA } \\
\hline rag2-KRASG12D & David Langenau $^{19}$ & $\mathrm{~N} / \mathrm{A}$ \\
\hline rag2-eGFP & David Langenau & $\mathrm{N} / \mathrm{A}$ \\
\hline pLKO.1-shSIX1 KD5 (3'UTR) & Functional Genomics Core & TRCN0000015233 \\
\hline pLKO.1-shSIX1 KD6 (CDS) & Functional Genomics Core & TRCN0000015236 \\
\hline pLKO.1-shScramble & Addgene & 1864 \\
\hline \multicolumn{3}{|l|}{ Software and algorithms } \\
\hline FastQC & Babraham Bioinformatics & $\begin{array}{l}\text { https://www.bioinformatics.ba } \\
\text { braham.ac.uk/projects/ }\end{array}$ \\
\hline BBDuk & Joint Genome Institute & $\begin{array}{l}\text { http://jgi.doe.gov/data-and- } \\
\text { tools/bb-tools }\end{array}$ \\
\hline STAR & 65 & $\begin{array}{l}\text { http://code.google.com/p/rna- } \\
\text { star/ }\end{array}$ \\
\hline edgeR & 66 & $\begin{array}{l}\text { https://bioconductor.org/pack } \\
\text { ages/edgeR }\end{array}$ \\
\hline clusterProfiler & 67 & $\begin{array}{l}\text { https://bioconductor.org/pack } \\
\text { ages/clusterProfiler }\end{array}$ \\
\hline
\end{tabular}




\begin{tabular}{|c|c|c|}
\hline RCisTarget & 68 & $\begin{array}{l}\text { https://bioconductor.org/pack } \\
\text { ages/RcisTarget }\end{array}$ \\
\hline Bowtie2 (v.2.3.4.3) & 69 & $\begin{array}{l}\text { http://bowtie- } \\
\text { bio.sourceforge.net/bowtie2/i } \\
\text { ndex.shtml }\end{array}$ \\
\hline Samtools (v.1.11) & 70 & http://www.htslib.org/ \\
\hline Picard & Broad Institute & $\begin{array}{l}\text { http://broadinstitute.github.io/ } \\
\text { picard/ }\end{array}$ \\
\hline Bedtools & $\overline{71}$ & $\begin{array}{l}\text { https://github.com/arq5x/bedt } \\
\text { ools2/releases }\end{array}$ \\
\hline MACS2 & 72 & $\begin{array}{l}\text { https://pypi.org/project/MACS } \\
2 /\end{array}$ \\
\hline ChIPseeker & 73 & $\begin{array}{l}\text { https://bioconductor.org/pack } \\
\text { ages/ChIPseeker/ }\end{array}$ \\
\hline ngs.plot & 74 & $\begin{array}{l}\text { https://github.com/shenlab- } \\
\text { sinai/ngsplot }\end{array}$ \\
\hline deepTools & 75 & https://github.com/deeptools \\
\hline HOMER (v.4.11) & 76 & http://homer.ucsd.edu/homer/ \\
\hline Rank Ordering of Super Enhancers (ROSE) & 29,30 & $\begin{array}{l}\text { http://younglab.wi.mit.edu/sup } \\
\text { er_enhancer_code.html }\end{array}$ \\
\hline R version 3.6.3 & The R project & www.r-project.org \\
\hline Python version 3.8 & Python & https://www.python.org/ \\
\hline FIJI & ImageJ & http://imagej.nih.gov/ij \\
\hline Prism 9 & GraphPad & www.graphpad.com \\
\hline IGV 2.8.0 & Broad Institute & $\begin{array}{l}\text { https://software.broadinstitute } \\
\text {.org/software/igv/2.8.x }\end{array}$ \\
\hline Signature Scoring Algorithm (S-score) & 38 & In-house R scripts \\
\hline \multicolumn{3}{|l|}{ Other } \\
\hline Nunc LabTek Chamber Slide System & ThermoFisher & 154526PK \\
\hline
\end{tabular}

\section{Resource Availability}

\section{Lead Contacts}

620 Further information and requests for resources and reagents should be directed to and will be fulfilled by 621 the lead contacts, Heide L. Ford (heide.ford@cuanschutz.edu) and Kristin B. Artinger 622 (kristin.artinger@cuanschutz.edu)

\section{Materials availability}

625 This study did not generate new unique reagents. 


\section{Data and code availability}

Scripts and code generated during this study are available upon request. RNAseq and ChIPseq datasets generated in this paper are deposited on GEO (GSE173155). Clinical datasets analyzed in this study are provided in the Key Resources table.

\section{Method Details}

\section{Clinical RNAseq Datamining}

Clinical sarcoma expression data was obtained from the $\mathrm{NCl}$ Oncogenomics database managed by Dr. Javed Khan at the NIH. Clinical RMS RNAseq expression data was downloaded from the St. Jude PeCAN portal and Integrated Rhabdomyosarcoma Database.

\section{Zebrafish line maintenance}

Zebrafish lines used in this study were maintained in compliance with the University of Colorado Anschutz Medical Campus IACUC guidelines and policies. The six $1 b^{o z 1}$ mutant line used in this study was a generous gift from Dr. Sharon Amacher's lab and crossed as heterozygotes to generate wildtype, heterozygote, and mutant homozygote progeny. Fish were genotyped as described previously described ${ }^{11}$.

\section{Zebrafish ERMS Studies}

Zebrafish ERMS tumors were established using previously described methods by the Langenau Lab. rag2-kRASG12D and rag2-eGFP plasmids were linearized with Notl and purified using the Zymo Clean and Concentrator kit. Linearized DNA was diluted to a stock concentration of $100 \mathrm{ng} / \mu \mathrm{L}$ and injected with phenol-red dye into the single-cell stage of embryos for a final concentration of $5 \mathrm{pg} / \mathrm{embryo}$ per rag2 plasmid. Zebrafish tumor initiation events were recorded at 36 days post-injection and every week 
thereafter until 180 days. Tumor area was measured weekly using a Leica epifluorescent stereomicroscope along with body length to adjust for changes in basal growth of fish.

\section{Zebrafish in situ hybridization on zRMS tissue}

Zebrafish tumor and normal muscle control tissues were fixed in 4\% PFA for 2 hours at room temperature

(RT), rinsed with PBS, and embedded in $1.5 \%$ agar/5\% sucrose solution. Agar-sucrose tissue blocks were flash-frozen in liquid nitrogen and subsequently cryosectioned on a microtome. Frozen sections were defrosted for $1 \mathrm{~h}$ at RT then incubated overnight at $70^{\circ} \mathrm{C}$ in six $1 \mathrm{~b}$ probe (provided by Vladimir Korzh, Institute of Medical and Cellular Biology, A*STAR, Proteos, Singapore) diluted $1 \mu \mathrm{g} / \mathrm{ml}$ in hybridization buffer (1X SSC buffer, 50\% formamide, 10\% dextran sulfate, 1mg/ml yeast tRNA, 1X Denhardt's).

Sections were then washed $3 \times 30 \mathrm{~min}$ at $70^{\circ} \mathrm{C}$ (Wash: $1 \mathrm{X}$ Saline Sodium Citrate (SSC) buffer, $50 \%$ formamide, 0.1\% Tween-20) followed by 3x10min at RT in MABT (1X maleic acid buffer, 20\% Tweenethanol solutions, cleared in xylene and coverslipped in Permount.

\section{Whole-mount zebrafish embryo in situ hybridization}

672 Whole-mount RNA in situ hybridization in zebrafish embryos was performed as previously described ${ }^{77}$. 673 DIG-conjugated antisense probes (gifts from Simon Hughes' lab) were T7 or T3 transcribed for pax3a, 674 myod1, and myogenin from pCS2+ backbone plasmids. Post-hoc genotyping of ISH-stained embryos 675 was performed by incubating single embryos in $300 \mathrm{mM} \mathrm{NaCl}$ overnight at $65^{\circ} \mathrm{C}$ to reverse crosslinks. 
DNA was purified from each embryo by phenol-chloroform extraction and genotyped as described previously ${ }^{11}$.

\section{Cell Culture and Cell lines}

680 FP-RMS and FN-RMS cell lines used in this study were a generous donation from Dr. Mark Hatley. Cell 681 lines manipulated in this study (SMS-CTR and RD) were maintained at $37^{\circ} \mathrm{C}$ and $5 \% \mathrm{CO}_{2}$ in Dulbecco's 682 Modified Eagle Medium (DMEM) supplemented with 10\% FBS and 1\% penicillin/streptomycin. Cell lines 683 were tested for mycoplasma (Lonza MycoAlert) at least twice per year and only mycoplasma-negative 684 cell lines were used in this study. All cell lines were STR authenticated by the University of Colorado 685 Cancer Center Tissue Culture shared resource.

Stable SIX1 KD was achieved in SMS-CTR and RD cell lines by lentiviral transduction of two pLKO.1688 derived shRNAs targeting the SIX1 CDS, subsequently denoted throughout the text as SIX1 KD5 and packaging plasmids. Viral particles were collected from 293T cells 48-hours post-transfection, passed $10 \%$ FBS/DMEM for 1 week and maintained in half the aforementioned puromycin dose for remaining experiments.

\section{IncuCyte Cell Growth Assay}

698 RMS cell growth was measured on an IncuCyte Zoom (Essen Bioscience) Live-Cell Analysis platform. 699 For cell growth, cells were plated at a concentration of 2500 cells/well in a 96 -well plate and imaged every 70012 hours with a 4 X objective. Cell growth was measured by percent confluence and results presented in 701 this study are normalized to percent confluence at time point zero (\% Confluence to Baseline). 


\section{qRT-PCR}

Cells were harvested for RNA using the Zymo Direct-zol RNA isolation kit and cDNA was synthesized using the Bio-rad iScript reverse transcription kit following manufacturer's instructions. Real-time qPCR was performed using Bio-rad ssoFast Evagreen supermix on a Biorad CFX96 qPCR instrument. SYBR primers used in this study are detailed in Supplementary Table S1.1.

Zebrafish tissues were snap-frozen in Trizol reagent, allowed to thaw, and homogenized using a plastic pestle. Homogenized tissue was then harvested for RNA using the Zymo Direct-zol kit. cDNA was synthesized using the ThermoFisher Verso cDNA Synthesis kit and qPCR reactions were performed using Taqman Gene Expression Master mix on an Applied Biosystems StepOnePlus instrument. Taqman probes used in this study are detailed in Supplemental Table S1.3.

\section{Western Blotting}

716 Whole cell protein extracts were harvested by lysing cells in RIPA buffer treated with protease inhibitors and further lysed via sonification. $20-50 \mu \mathrm{g}$ of whole cell lysates were boiled with sample buffer and run through a $10 \%$ polyacrylamide gel. After PAGE gel electrophoresis, gels were transferred onto PVDF membranes, blocked in 5\% Milk/TBST, and incubated with primary antibodies diluted in 5\%BSA/TBST

overnight at $4^{\circ} \mathrm{C}$. Blots were incubated with $\mathrm{HRP}$-conjugated secondary antibodies raised against primary antibody species at a 1:1000 dilution and chemiluminescence detected with Pierce ECL Western Blotting substrate. Chemiluminescence was imaged using an OdysseyFc imaging instrument. Between all antibody incubations, blots were washed with 1X TBST.

\section{Immunocytochemistry}

Cells were plated on 4-well chamber slide and fixed in 4\% PFA/PBS for 10 minutes and permeabilized 
serum/PBST for one hour and incubated in primary antibody solution overnight. The following day, chamber slides were incubated with appropriate fluorophore-conjugated secondary antibodies and mounted with Vectashield mounting medium with DAPI counterstain. All washes between incubation steps were performed with 1X PBS. Mounted slides were imaged on an Olympus BX51 fluorescence microscope. For phH3 and myHC stains, staining was quantified by dividing the number of positively stained cells by the total number of nuclei per field of view. Multinucleated events or fusion indices were quantified by counting the number of nuclei enclosed within a single positively stained myHC unit. For all immunocytochemistry stains, data is represented as image measurements taken over at least three independent experiments with two or more biological replicates per experiment, and two or more fields of view per biological replicate.

\section{Mouse Studies}

All mouse studies were performed in 6-8 week old immunodeficient NOD/SCID $\gamma$ (NSG) of mixed genders. For mouse xenograft experiments, $2 \times 10^{5}$ cells suspended in a $200 \mu \mathrm{L} 1: 1$ matrigel:1X PBS suspension were subcutaneously injected into either the left or right flank of the mouse, with each mouse receiving both a shScramble and SIX1 KD injection on one flank. Tumor growth was measured weekly for 12 weeks using calipers or until tumors surpassed a tumor volume of $1000 \mathrm{~mm}^{3}\left(1 \mathrm{~cm}^{3}\right)$. All animal studies were performed according to protocols approved by the University of Colorado Institutional Animal Care and Use Committee.

\section{Immunohistochemistry}

For zRMS studies, tumor-burdened fish were euthanized in ice-water, fixed in $4 \% \mathrm{PFA}$ overnight at $4^{\circ} \mathrm{C}$, washed in PBS for 24 hours, decalcified in 20\% EDTA pH 8.0 for 24 hours, dehydrated in $70 \% \mathrm{EtOH}$, and paraffin-embedded. Paraffin-embedded tissues were cut into 10-15 $\mu$ m thick sections and stained with $\mathrm{H} \& \mathrm{E}$ or further processed for antibody staining. 
For mouse xenografts following dissection, mouse tumor tissue was fixed in 4\% PFA overnight, washed in PBS for 24 hours, and dehydrated in $70 \% \mathrm{EtOH}$ prior to paraffin-embedment. For all downstream IHC stains (zRMS, mouse xenograft, human tissue array), slides were de-paraffinized and retrieved in either pH6 (Six1, myHC) or pH9 (Pax7) Tris/EDTA buffer. Slides were then peroxidase blocked with 3\% hydrogen peroxide (in methanol) for $10 \mathrm{~min}$, blocked in serum-free blocking reagent (DAKO) and incubated with primary antibodies for $1 \mathrm{hr}$ at room temperature. Appropriate species' secondary antibodies were then incubated for $30 \mathrm{~min}$ and developed with DAB stain for $10 \mathrm{~min}$ and counterstained with hematoxylin for another 8 min.

\section{RNA sequencing and Analysis}

Total RNA was isolated from SMS-CTR cells using the Zymo Direct-zol RNA Miniprep Kit and RNA integrity confirmed using TapeStation analysis. shScramble and SIX1 KD SMS-CTR RNA samples were submitted as biological triplicates except for SIX1 KD6 which was submitted as biological duplicates on account of its marked proliferative defects. 100ng of total RNA per sample was used to construct PolyAselected RNA libraries for RNAseq and sequenced using paired end reads with 150 cycles on an Illumina NovaSEQ 6000 instrument. Read QC was performed using fastqc and reads were trimmed with BBDuk to remove lllumina adapter sequences and the first 12 bases on the 5' ends. Trimmed fastqc files were aligned to the hg38 human reference genome and aligned counts per gene were quantified using STAR ${ }^{65}$. Differential gene analysis was performed using the edgeR package ${ }^{66}$. Gene Set Enrichment Analysis (GSEA) was performed under default settings using the clusterProfiler R package gseaplot function ${ }^{67}$. Normalized counts (CPM) were converted to $z$-scores prior to plotting and heatmaps were created using the pheatmaps $\mathrm{R}$ package (https://CRAN.R-project.org/package=pheatmap).

\section{Chromatin Immunoprecipitation (ChIPseq)}

Human cells along with spike-in Drosophila S2 cells at a 1:10 ratio with human cells were fixed in 1\% formaldehyde diluted in growth media for an incubation time of 15 minutes. Crosslinking was quenched 
with the direct addition of $1 \mathrm{M}$ Tris $\mathrm{pH} 7.5$ and shaking for 15 minutes. Cells were gently scraped off plates, pelleted by centrifugation, washed in cold PBS and centrifuged again. Cell pellets were snap frozen in liquid nitrogen and nuclei were extracted from cell pellets (Sigma Nuclei Isolation Kit \#NUC-101). Chromatin was fragmented in sonication buffer (50mM HEPES pH 7.5, $140 \mathrm{mM} \mathrm{NaCl}, 1 \mathrm{mM}$ EDTA, $1 \mathrm{mM}$ EGTA, $1 \%$ Triton-X, $0.1 \%$ Sodium deoxycholate, $0.1 \%$ SDS) supplemented with protease inhibitor cocktail using a Branson digital sonifier instrument at $4^{\circ} \mathrm{C}$ with the following settings: 7 cycles of $30 \mathrm{~s}$ ON and $1 \mathrm{~m}$ OFF sonification at $50 \%$ intensity. Chromatin lysates were incubated with $10 \mu \mathrm{g}$ antibody-bound Dynabeads (Dynabeads: Fisher Scientific \#14-311-D; see supplemental materials for antibody information) overnight and subsequently washed in buffers of increasing stringency: $2 X$ sonication buffer, $1 \mathrm{X}$ high salt sonication buffer (sonication buffer with $500 \mathrm{mM} \mathrm{NaCl}), 1 \mathrm{X} \mathrm{LiCl}$ buffer $(20 \mathrm{mM}$ Tris pH 8.0, $1 \mathrm{mM}$ EDTA, $250 \mathrm{mM} \mathrm{LiCl}, 0.5 \%$ NP-40, $0.5 \%$ sodium deoxycholate), and $1 \mathrm{X}$ TE pH 8.0. Immunocomplexes were eluted in 1\% SDS/TE buffer and transferred to Lobind DNA tubes (Eppendorf $\# 13-698-790)$ at $65^{\circ} \mathrm{C}$ for 30 minutes and crosslinks were reversed overnight by incubating samples at $65^{\circ} \mathrm{C}$. RNA and protein were digested by the addition of RNase and Proteinase $\mathrm{K}$, and DNA fragments were finally purified using phenol-chloroform. ChIPseq libraries were assembled using the KAPA HyperPrep ChIP library kit following manufacturer's settings and were sequenced on an Illumina Nextseq500 machine.

\section{CUT\&RUN}

500,000 cells/sample were harvested by scraping and were resuspended and washed twice in wash buffer supplemented with protease inhibitor cocktail (20mM HEPES pH 7.5, $150 \mathrm{mM} \mathrm{NaCl}, 0.5 \mathrm{mM}$ Spermidine). Cells were adsorbed onto activated Concavalin A beads for 10 minutes and then incubated with antibodies $\mathrm{O} / \mathrm{N}$ at $4^{\circ} \mathrm{C}$. After antibody incubation, unbound antibodies were washed away with cold Digitonin buffer (wash buffer $+0.01 \%$ Digitonin) and pAG-MNase was added to each sample to produce chromatin fragments under targets for 10 minutes at room temperature. Cells were then cooled to $0^{\circ} \mathrm{C}$ 
805

806

and incubated with ice cold $100 \mathrm{mM} \mathrm{CaCl}_{2}$ for 2 hours at $4^{\circ} \mathrm{C}$. MNase digestion was terminated with the addition of a master mix of STOP buffer (340mM NaCl, 20mM EDTA, 4mM EGTA, 50ug/mL RNaseA, $50 \mu \mathrm{g} / \mathrm{mL}$ Glycogen) and $0.5 \mathrm{ng} / \mathrm{ul}$ E.coli spike-in DNA and incubated for 10 minutes at $37^{\circ} \mathrm{C}$. DNA was finally purified using a column purification kit and subsequently used for library assembly. Antibody concentrations: 1:100 for rabbit IgG and 1:50 for MYOD1. CUT\&RUN libraries were assembled using the NEBNext II Ultra Library Prep kit) and dual-index primers following manufacturer protocols. Library size distribution was assessed by TapeStation and libraries were subsequently used for CUT\&RUN qPCR.

\section{ChIPseq Analysis}

The quality of the fastq files was accessed using FastQC (https://www.bioinformatics.babraham.ac.uk/projects/) and MultiQC ${ }^{78}$. Illumina adapters and low-quality reads were filtered out using BBDuk (http://jgi.doe.gov/data-and-tools/bb-tools). Bowtie2 (v.2.3.4.3) was used to align the sequencing reads to the hg38 reference human genome and to the dm6 drosophila reference genome ${ }^{69}$. Samtools (v.1.11) was used to select the mapped reads (samtools view - $b-q$ 30) and sort the bam files ${ }^{70}$. PCR duplicates were removed using Picard MarkDuplicates tool (http://broadinstitute.github.io/picard/). The normalization ratio of each sample was calculated by dividing the total number of mapped reads mapping to the Drosophila genome of each sample by the total number of mapped reads mapping to the Drosophila genome of the sample with the lowest number of reads. Using the normalization ratio, random sub-sampling of the reads was performed using samtools view hs. Bedtools genomecov was used to create bedgraph files from the bam files ${ }^{71}$. Peaks were called using MACS2 (v2.1.2) with default parameters for narrow peaks (--gsize hs --qvalue 0.01$)^{72}$. Average profiles were generated using ngs.plot ${ }^{74}$ and heatmaps were generated using bigwig files with deepTools ${ }^{75}$. ChIP peaks were annotated using the ChIPseeker $\mathrm{R}$ package ${ }^{73}$. Super-enhancers were identified using the Ranking Ordering of Super-Enhancer (ROSE) algorithm using default parameters ${ }^{29,30}$ and hockey stick plots were generated in R. ChIPseq track figures were generated using the Washington University Epigenome Browser ${ }^{79}$. 


\section{Statistical Analysis}

833 For all cell line experiments, experiments were performed in at least three independent biological 834 experiments with biological replicates and reported in this manuscript as a composite of these biological 835 replicates. Therefore, when applicable, error bars for all figures including both cell line and animal 836 experiments depict standard error of the mean (SEM). For all zebrafish experiments, an unpaired two837 sided Student's $t$-test was used to compare wildtype/control measurements to that of $\operatorname{six}_{1} b^{-/}$sibling or 838 appropriately age-matched tumor tissue. For all cell line data, statistical differences between control and 839 SIX1 KD conditions were measured using an unpaired two-sided Student's $t$-test, unless specified 840 otherwise in the figure legends. For animal experiments (both zebrafish and mouse) comparing tumor 841 growth over time (Figure 2 \& 3), tumor growth data were fitted to a Longitudinal Mixed Effect model and 842 tumor growth was compared between shScramble and SIX1 KD mouse groups or wildtype and six1b 843 mutant fish groups. Throughout this manuscript, all $p$-values are reported as is on figures or in figure 844 legends.

\section{Acknowledgments:}

847 We would like to thank Dr. Brian Abraham for assistance with ChIPseq analysis and Erin Binne, Virginia 848 Ware, and Taylor Hotz for their assistance throughout this project. We would also like to acknowledge a 849 number of undergraduates whose help over the years contributed to this body of work: Julia Torline, 850 Aaron Clark, Oscar Yip, and Hope Eden. This work was generously supported by NIH grants 851 R21CA201809 (to H.L.F and K.B.A), R01CA224867 (to H.L.F), R01CA183874 (to P.J), K08CA245251 852 (to A.D.D), the Alex's Lemonade Stand Foundation Innovation Award (to H.L.F), the CU Cancer Center 853 Molecular and Cellular Oncology Pilot Grant P30CA046934 (to H.L.F, K.B.A, and P.J), and training 854 fellowships T32GM763538 and TL1TR001081 (to J.Y.H). This work utilized the Cell Technologies, 855 Functional Genomics, Pathology, and Biostatistics and Bioinformatics Shared Resource supported by 856 P30CA046934 (to the University of Colorado Cancer Center). Research reported was also supported by 
857 the NIH/NCI P30CA021765 (to St. Jude Children's Research Hospital Comprehensive Cancer Center), 858 CureSearch for Children's Cancer Foundation, Rally Foundation for Childhood Cancer Research, and 859 the American Lebanese Syrian Associated Charities (to A.D.D and S.N).

860

861 Diversity and Inclusion Statement:

862 We worked to ensure sex balance/diversity in experimental samples through the selection of the cell 863 lines, selection of non-human subjects, and selection of the genomic datasets (all which contained both 864 male and female samples). The author list of this paper includes contributors from the location where 865 the research was conducted who participated in the data collection, design, analysis, and/or 866 interpretation of the work.

867

868 Declaration of Interests:

869 J.C.C is a co-founder of PrecisionProfile. H.L.F is a co-founder of Sieyax, LLC. 


\section{References:}

872 1. Rekhi, B. et al. Clinicopathologic features of 300 rhabdomyosarcomas with emphasis upon differential expression of skeletal muscle specific markers in the various subtypes: A single institutional experience.

5. Yang, Z. et al. MyoD and E-protein heterodimers switch rhabdomyosarcoma cells from an arrested myoblast phase to a differentiated state. Genes Dev. 23, 694-707 (2009).

6. Cheyette, B. N. R. et al. The drosophila sine oculis locus encodes a homeodomain-containing protein required for the development of the entire visual system. Neuron 12, 977-996 (1994).

7. Laclef, C. et al. Altered myogenesis in Six1-deficient mice. 2239-2252 (2003). doi:10.1242/dev.00440

8. Grifone, R. et al. Six1 and Six4 homeoproteins are required for Pax3 and Mrf expression during myogenesis in the mouse embryo. (2004). doi:10.1242/dev.01773

9. Lin, C. Y. et al. The transcription factor Six1a plays an essential role in the craniofacial myogenesis of zebrafish. Dev. Biol. 331, 152-166 (2009).

10. Nord, H., Nygård Skalman, L. \& von Hofsten, J. Six1 regulates proliferation of Pax7-positive muscle progenitors in zebrafish. J. Cell Sci. 126, 1868-80 (2013).

11. Talbot, J. C. et al. Muscle precursor cell movements in zebrafish are dynamic and require six- family genes. (2019). doi:10.1242/dev.171421

12. Zammit, P. S. Function of the myogenic regulatory factors Myf5, MyoD, Myogenin and MRF4 in skeletal muscle, satellite cells and regenerative myogenesis. Semin. Cell Dev. Biol. 72, 19-32 (2017). Differentiation-Specific Transcription in Myogenesis. Mol. Cell 47, 457-468 (2012). 
14. Cao, Y. et al. Genome-wide MyoD Binding in Skeletal Muscle Cells: A Potential for Broad Cellular Reprogramming. Dev. Cell 18, 662-674 (2010).

15. Spitz, F. et al. Expression of myogenin during embryogenesis is controlled by Six/sine oculis homeoproteins through a conserved MEF3 binding site. Proc. Natl. Acad. Sci. U. S. A. 95, 14220-14225 (1998).

16. Santolini, M. et al. MyoD reprogramming requires Six1 and Six4 homeoproteins: genome-wide cisregulatory module analysis. Nucleic Acids Res. 44, 8621-8640 (2016).

17. Relaix, F. et al. Six Homeoproteins Directly Activate Myod Expression in the Gene Regulatory Networks That Control Early Myogenesis. PLoS Genet. 9, (2013).

18. Dharia, N. V. et al. A first-generation pediatric cancer dependency map. Nat. Genet. (2021). doi:10.1038/s41588-021-00819-w

19. Langenau, D. M. et al. Effects of RAS on the genesis of embryonal rhabdomyosarcoma. Genes Dev. 21, 1382-1395 (2007).

20. Sokolowski, E., Turina, C. B., Kikuchi, K., Langenau, D. M. \& Keller, C. Proof-of-concept rare cancers in drug development : the case for rhabdomyosarcoma. 1-13 (2013). doi:10.1038/onc.2013.129

21. Hinson, A. R. P. et al. Human Rhabdomyosarcoma Cell Lines for Rhabdomyosarcoma Research: Utility and Pitfalls. Front. Oncol. 3, 1-12 (2013).

22. Altevogt, P., Doberstein, K. \& Fogel, M. L1CAM in human cancer. Int. J. Cancer 138, 1565-1576 (2016).

23. Katoh, Y. \& Katoh, M. Hedgehog signaling, epithelial-to-mesenchymal transition and miRNA. Int. J. Mol. Med. 22, 271-275 (2008).

24. Lo, H. W. et al. Epidermal growth factor receptor cooperates with signal transducer and activator of transcription 3 to induce epithelial-mesenchymal transition in cancer cells via up-regulation of TWIST gene expression. Cancer Res. 67, 9066-9076 (2007).

25. Subramanian, A. et al. Gene set enrichment analysis: A knowledge-based approach for interpreting genome-wide expression profiles. Proc. Natl. Acad. Sci. U. S. A. 102, 15545-15550 (2005).

26. Micalizzi, D. S. et al. The Six1 homeoprotein induces human mammary carcinoma cells to undergo epithelial-mesenchymal transition and metastasis in mice through increasing TGF-beta signaling. J. Clin. Invest. 119, 2678-90 (2009). 
27. Mccoy, E. L. et al. Six1 expands the mouse mammary epithelial stem / progenitor cell pool and induces mammary tumors that undergo epithelial- mesenchymal transition. 119, 2663-2677 (2009).

28. Leikina, E. et al. Myomaker and Myomerger Work Independently to Control Distinct Steps of Membrane Remodeling during Myoblast Fusion. Dev. Cell 1-14 (2018). doi:10.1016/j.devcel.2018.08.006

29. Whyte, W. A. et al. Master transcription factors and mediator establish super-enhancers at key cell identity genes. Cell 153, 307-319 (2013).

30. Lovén, J. et al. Selective inhibition of tumor oncogenes by disruption of super-enhancers. Cell 153, 320334 (2013).

31. Noguchi, Y. T. et al. Cell-autonomous and redundant roles of Hey1 and HeyL in muscle stem cells: HeyL requires HeS1 to bind diverse DNA sites. Dev. 146, 1-12 (2019).

32. Qamar, L. et al. Specificity and prognostic validation of a polyclonal antibody to detect Six1 homeoprotein in ovarian cancer. Gynecol. Oncol. 125, 451-457 (2012).

33. Shi, J. et al. Requirement of the fusogenic micropeptide myomixer for muscle formation in zebrafish. Proc. Natl. Acad. Sci. U. S. A. 114, 11950-11955 (2017).

34. Skene, P. J. \& Henikoff, S. An efficient targeted nuclease strategy for high-resolution mapping of DNA binding sites. Elife 6, e21856 (2017).

35. Li, S. et al. Twist2 amplification in rhabdomyosarcoma represses myogenesis and promotes oncogenesis by redirecting MyoD DNA binding. Genes Dev. 33, 626-640 (2019).

36. Pomella, S. et al. Interaction between SNAI2 and MYOD enhances oncogenesis and suppresses differentiation in Fusion Negative Rhabdomyosarcoma. Nat. Commun. 12, 1-15 (2021).

37. Choi, I. Y. et al. Transcriptional landscape of myogenesis from human pluripotent stem cells reveals a key role of TWIST1 in maintenance of skeletal muscle progenitors. Elife 9, 1-27 (2020).

38. Hsiao, T. H. et al. Utilizing-score to identify oncogenic pathways of cholangiocarcinoma. Transl. Cancer Res. 2, 6-17 (2013).

39. Liu, Z. et al. CASZ1 induces skeletal muscle and rhabdomyosarcoma differentiation through a feedforward loop with MYOD and MYOG. Nat. Commun. 11, (2020).

40. Hayes, M. N. et al. Vangl2/RhoA Signaling Pathway Regulates Stem Cell Self-Renewal Programs and Growth in Rhabdomyosarcoma. Cell Stem Cell 22, 414-427.e6 (2018). 
41. Tenente, I. M. et al. Myogenic regulatory transcription factors regulate growth in rhabdomyosarcoma. Elife 6, (2017).

42. Phelps, M. P., Bailey, J. N., Vleeshouwer-Neumann, T. \& Chen, E. Y. CRISPR screen identifies the NCOR/HDAC3 complex as a major suppressor of differentiation in rhabdomyosarcoma. Proc. Natl. Acad. Sci. U. S. A. 113, 15090-15095 (2016).

43. An, Y. et al. A Molecular Switch Regulating Cell Fate Choice between Muscle Progenitor Cells and Brown Adipocytes. Dev. Cell 41, 382-391.e5 (2017).

44. Rudnicki, M. A. et al. MyoD or Myf-5 is required for the formation of skeletal muscle. Cell $75,1351-1359$ (1993).

45. Berti, F. et al. Time course and side-by-side analysis of mesodermal, pre-myogenic, myogenic and differentiated cell markers in the chicken model for skeletal muscle formation. J. Anat. 227, 361-382 (2015).

46. O’Brien, J. H., Hernandez-Lagunas, L., Artinger, K. B. \& Ford, H. L. MicroRNA-30a regulates zebrafish myogenesis through targeting the transcription factor Six1. J. Cell Sci. 127, 2291-2301 (2014).

47. Yu, Y. et al. Expression profiling identifies the cytoskeletal organizer ezrin and the developmental homeoprotein Six-1 as key metastatic regulators. Nat. Med. 10, 175-181 (2004).

48. Yu, Y., Davicioni, E., Triche, T. J. \& Merlino, G. The Homeoprotein Six1 Transcriptionally Activates Multiple Protumorigenic Genes but Requires Ezrin to Promote Metastasis. 1982-1989 (2006). doi:10.1158/00085472.CAN-05-2360

49. Grand, F. Le et al. Six1 regulates renewal during skeletal muscle regeneration. J. Cell Biol. 198, 815-832 (2012).

50. Bessarab, D. A., Chong, S.-W., Srinivas, B. P. \& Korzh, V. Six1a is required for the onset of fast muscle differentiation in zebrafish. Dev. Biol. 323, 216-228 (2008).

51. McCoy, E. L. et al. Six1 expands the mouse mammary epithelial stem/progenitor cell pool and induces mammary tumors that undergo epithelial-mesenchymal transition. J. Clin. Invest. 119, 2663-77 (2009).

52. Coletta, R. D. et al. The Six1 homeoprotein stimulates tumorigenesis by reactivation of cyclin A1. Proc. Natl. Acad. Sci. U. S. A. 101, 6478-6483 (2004).

53. Lee, E. J. et al. Establishment of stably expandable induced myogenic stem cells by four transcription 
factors. Cell Death Dis. 9, (2018).

54. Kingsbury, T. J., Kim, M. J. \& Civin, C. I. Regulation of cancer stem cell properties by SIX1, a member of the PAX-SIX-EYA-DACH network. Advances in Cancer Research 141, (Elsevier Inc., 2019).

55. Heinz, S., Romanoski, C. E., Benner, C. \& Glass, C. K. The selection and function of cell type-specific enhancers. Nat. Rev. Mol. Cell Biol. 16, 144-154 (2015).

56. Khan, J. et al. cDNA microarrays detect activation of a myogenic transcription program by the PAX3-FKHR fusion oncogene. Proc. Natl. Acad. Sci. 96, 13264-13269 (1999).

57. Gryder, B. E. et al. Miswired Enhancer Logic Drives a Cancer of the Muscle Lineage. iScience 23, (2020).

58. Gryder, B. E. et al. Histone hyperacetylation disrupts core gene regulatory architecture in rhabdomyosarcoma. Nat. Genet. 51, 1714-1722 (2019).

59. Gryder, B. E. et al. PAX3-FOXO1 establishes myogenic super enhancers and confers BET bromodomain vulnerability. Cancer Discov. 7, 884-899 (2017).

60. Farabaugh, S. M., Micalizzi, D. S., Jedlicka, P. \& Ford, H. L. Eya2 is Required to Mediate the ProMetastatic Functions of Six1 via the Induction of TGF- $\beta$ Signaling, Epithelial-to-Mesenchymal Transition, and Cancer Stem Cell Properties. Oncogene 31, 552-562 (2012).

61. Patrick, A. N. et al. Structure-function analyses of the human SIX1-EYA2 complex reveal insights into metastasis and BOR syndrome. Nat. Struct. Mol. Biol. 20, 447-53 (2013).

62. Li, X. et al. Eya protein phosphatase activity regulates Six1-Dach-Eya transcriptional effects in mammalian organogenesis. Nature 426, 247-254 (2003).

63. Zhou, H. et al. Identification of a small-molecule inhibitor that disrupts the SIX1/EYA2 complex, EMT, and metastasis. Cancer Res. 80, 2689-2702 (2020).

64. Hanna, J. A. et al. PAX3-FOXO1 drives miR-486-5p and represses miR-221 contributing to pathogenesis of alveolar rhabdomyosarcoma. Oncogene 37, 1991-2007 (2018).

65. Dobin, A. et al. STAR: ultrafast universal RNA-seq aligner. Bioinformatics 29, 15-21 (2013).

66. Robinson, M. D., McCarthy, D. J. \& Smyth, G. K. edgeR: a Bioconductor package for differential expression analysis of digital gene expression data. Bioinformatics 26, 139-140 (2010).

67. Yu, G., Wang, L. G., Han, Y. \& He, Q. Y. ClusterProfiler: An R package for comparing biological themes among gene clusters. Omi. A J. Integr. Biol. 16, 284-287 (2012). 
1011 68. Aibar, S. et al. SCENIC: single-cell regulatory network inference and clustering. Nat. Methods 14, 10831086 (2017).

69. Langmead, B. \& Salzberg, S. L. Fast gapped-read alignment with Bowtie 2. Nat. Methods 9, 357-359 (2012).

1015 70. Li, H. et al. The Sequence Alignment/Map format and SAMtools. Bioinformatics 25, 2078-2079 (2009).

1016 71. Quinlan, A. R. \& Hall, I. M. BEDTools: A flexible suite of utilities for comparing genomic features.

1017 Bioinformatics 26, 841-842 (2010).

1018 72. Zhang, Y. et al. Model-based analysis of ChIP-Seq (MACS). Genome Biol. 9, (2008).

1019 73. Yu, G., Wang, L. G. \& He, Q. Y. ChIP seeker: An R/Bioconductor package for ChIP peak annotation, $1020 \quad$ comparison and visualization. Bioinformatics 31, 2382-2383 (2015).

1021 74. Shen, L., Shao, N., Liu, X. \& Nestler, E. ngs.plot: Quick mining and visualization of next-generation sequencing data by integrating genomic databases. BMC Genomics 15, 284 (2014).

1023 75. Ramírez, F., Dündar, F., Diehl, S., Grüning, B. A. \& Manke, T. deepTools: a flexible platform for exploring deep-sequencing data. Nucleic Acids Res. 42, W187-W191 (2014).

76. Heinz, S. et al. Simple combinations of lineage-determining transcription factors prime cis-regulatory elements required for macrophage and B cell identities. Mol. Cell 38, 576-589 (2010).

1027 77. Thisse, B. et al. Spatial and Temporal Expression of the Zebrafish Genome by Large-Scale In Situ 1028 Hybridization Screening. in The Zebrafish: Genetics, Genomics, and Informatics 77, 505-519 (Academic Press, 2004).

1030 78. Ewels, P., Magnusson, M., Lundin, S. \& Käller, M. MultiQC: summarize analysis results for multiple tools 1031 and samples in a single report. Bioinformatics 32, 3047-3048 (2016). 


\section{Figure 1. SIX1 is overexpressed and predicted to be an essential gene in Rhabdomyosarcoma}

A

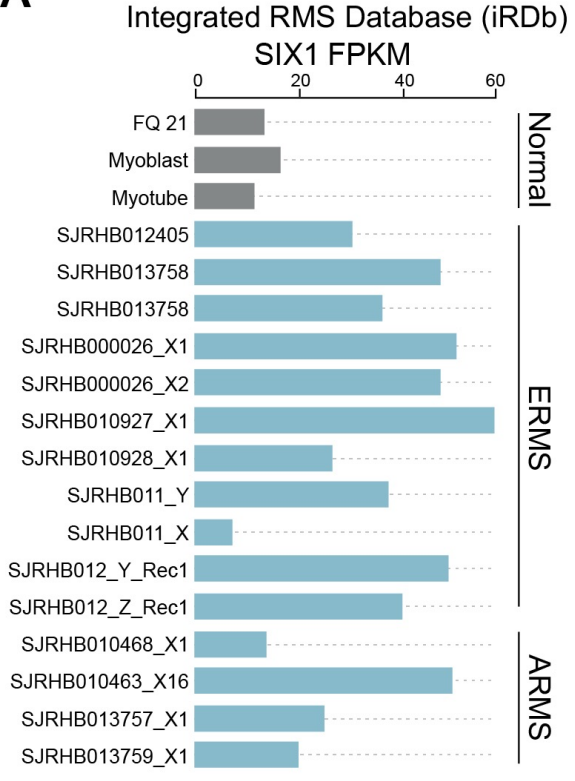

D

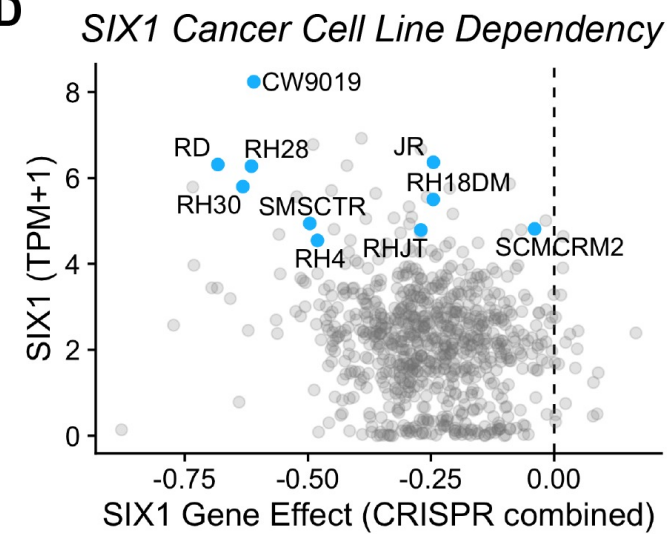

H

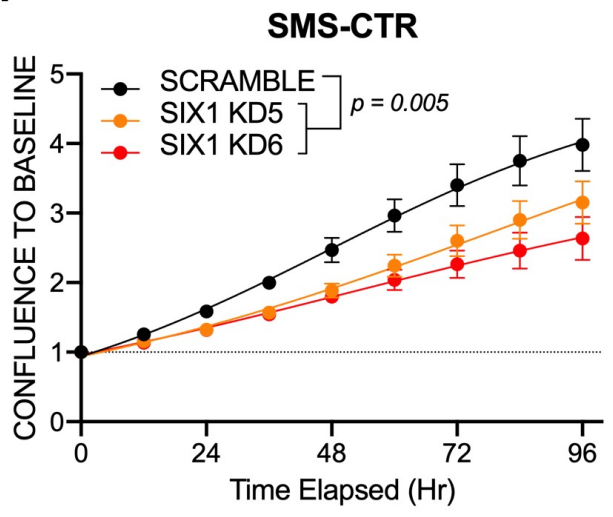

B

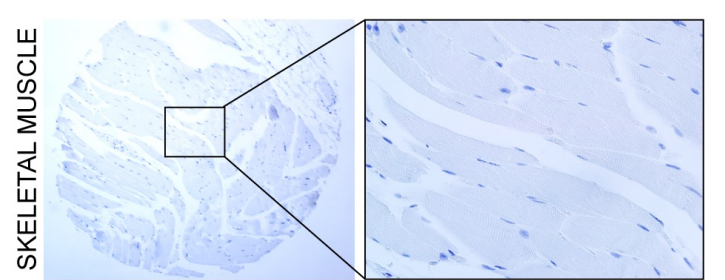

C
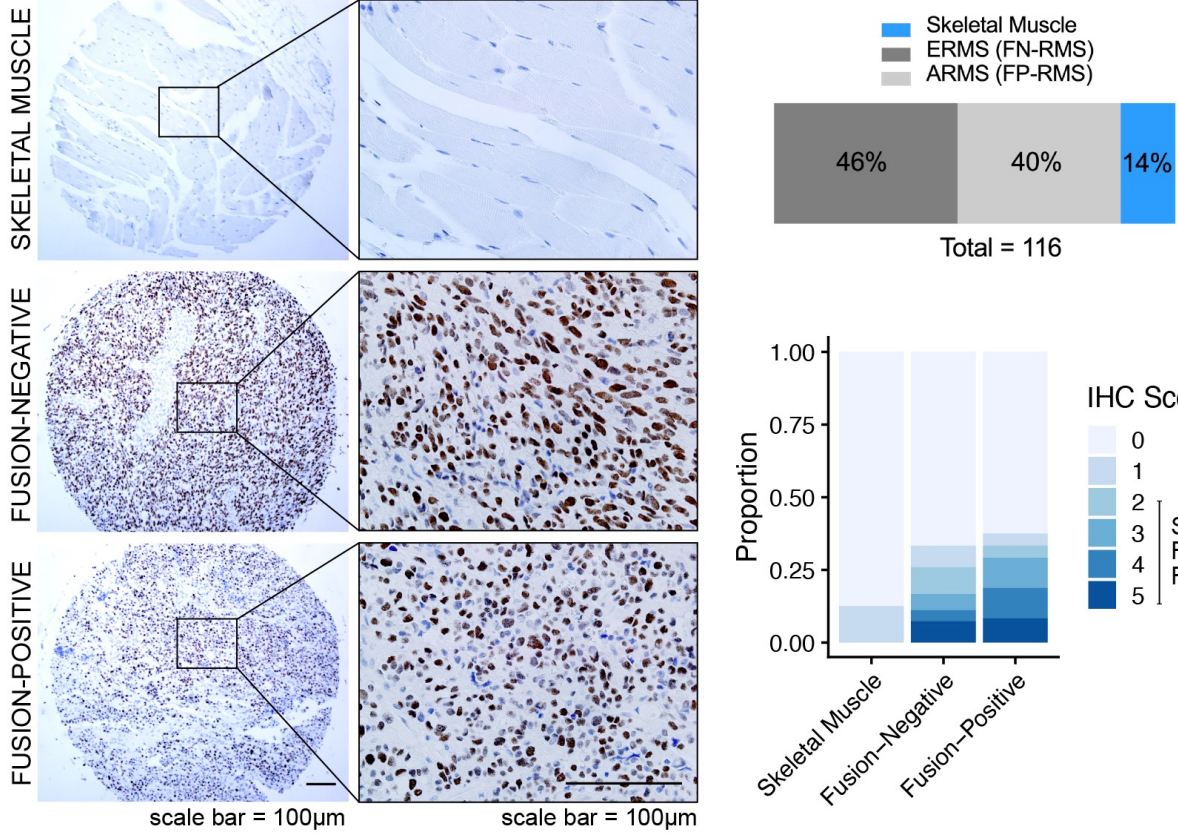

Total $=116$

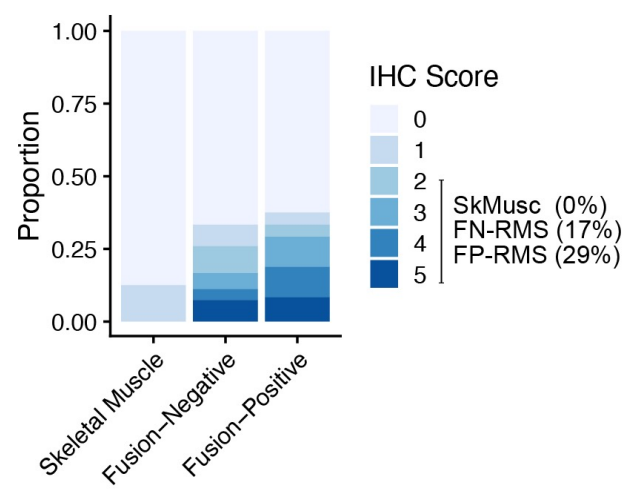

E

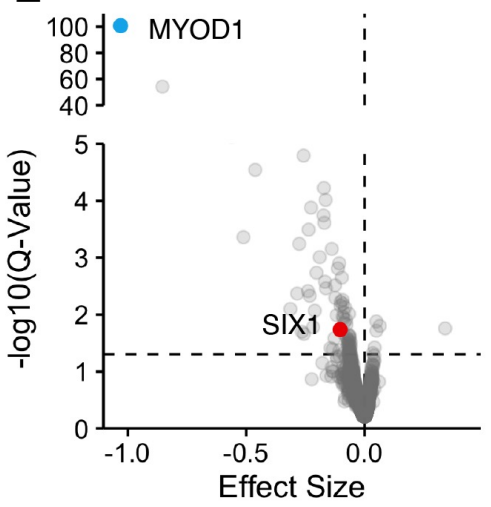

RD

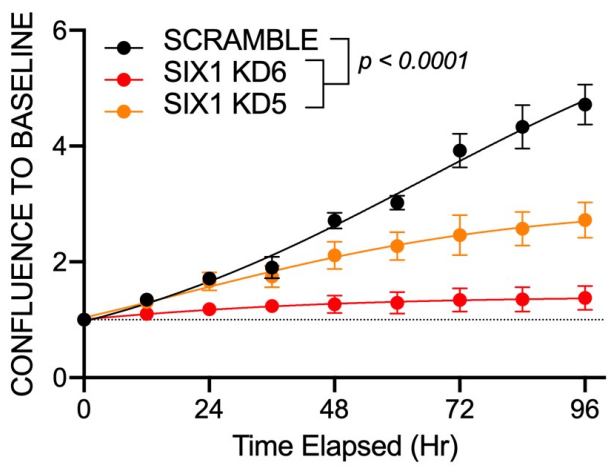

$\mathbf{F}$

G
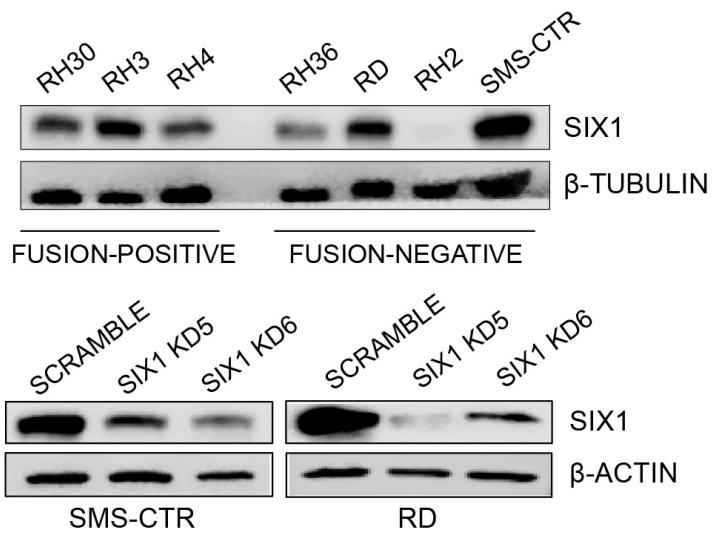

I

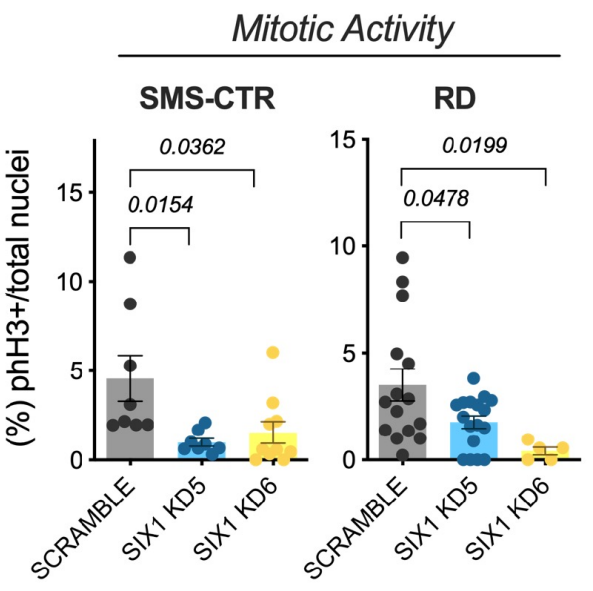




\section{Figure 2. six1b is required for zebrafish RMS tumor growth}

A

$\square$ Skeletal Muscle $\square$ zRMS Tumor

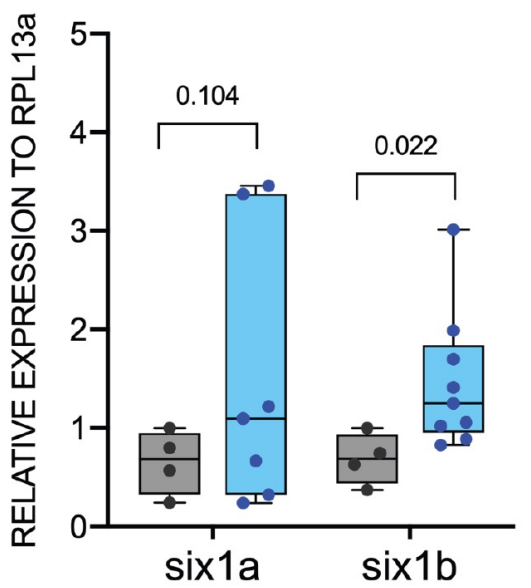

C

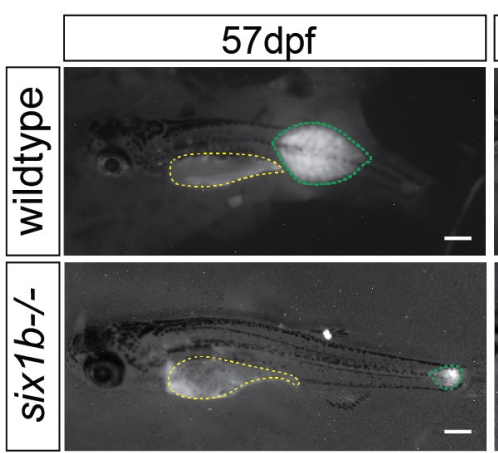

E

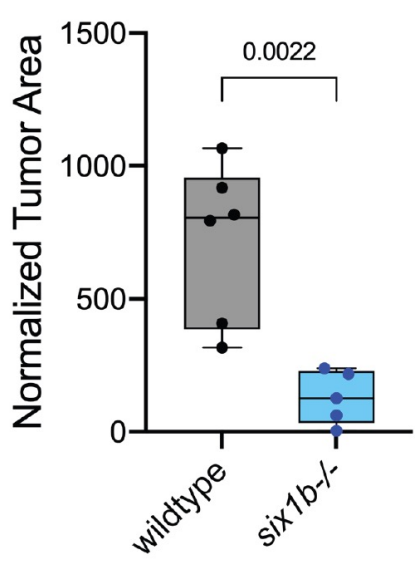

$\mathbf{F}$
B
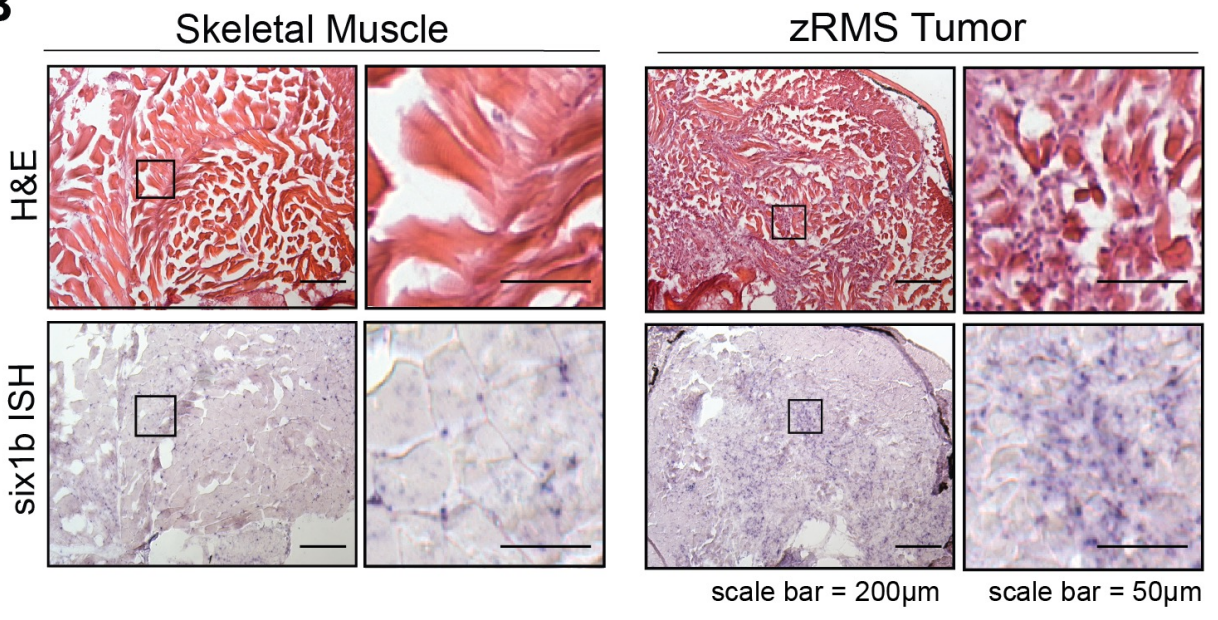

D
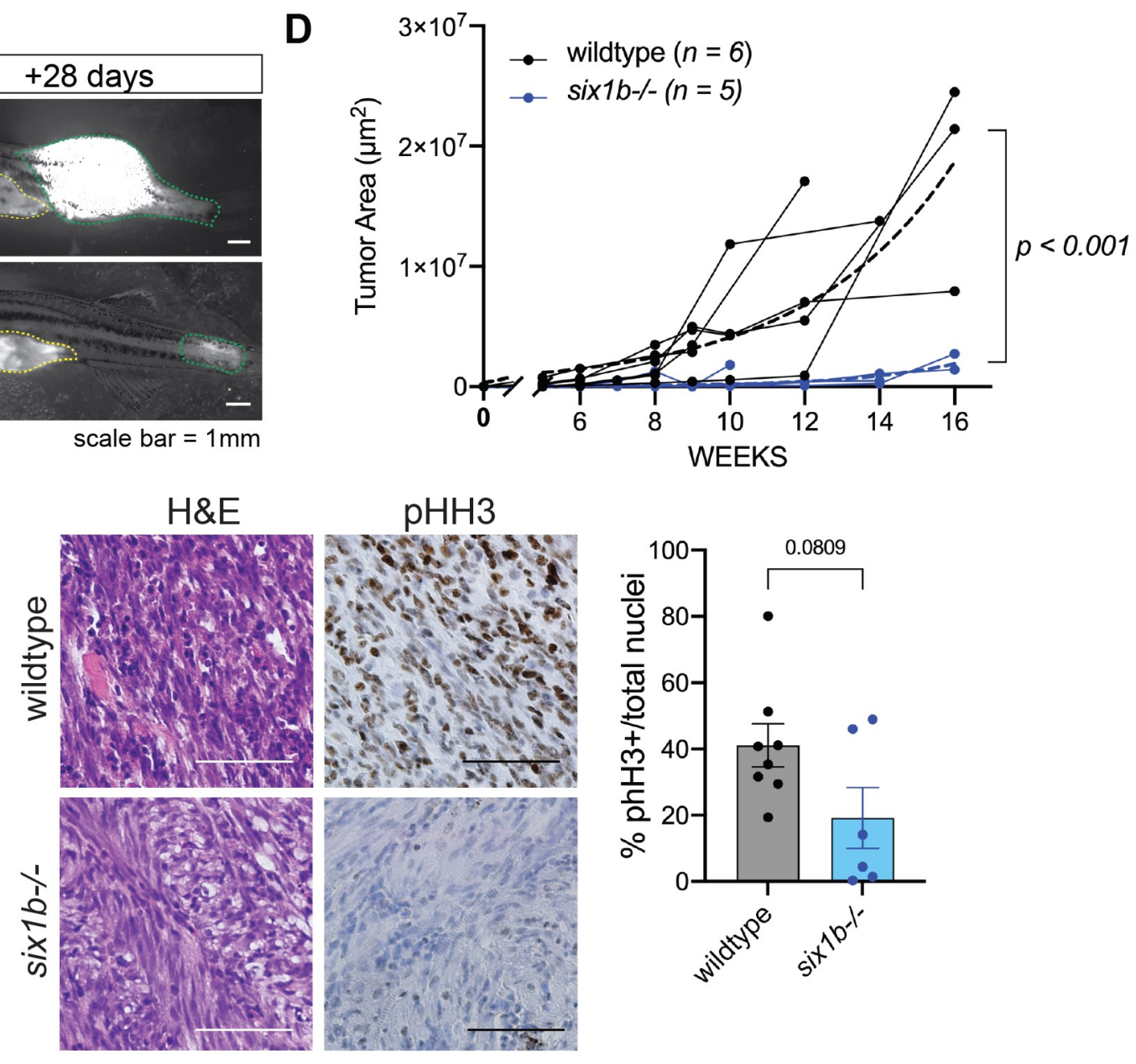

scale bar $=50 \mu \mathrm{m}$ 
bioRxiv preprint doi: https://doi.org/10.1101/2021.04.25.439216; this version posted May 16, 2021. The copyright holder for this preprint (which was not certified by peer review) is the author/funder. All rights reserved. No reuse allowed without permission.

\section{Figure 3. SIX1 knockdown inhibits human RMS tumor growth and progression}

A

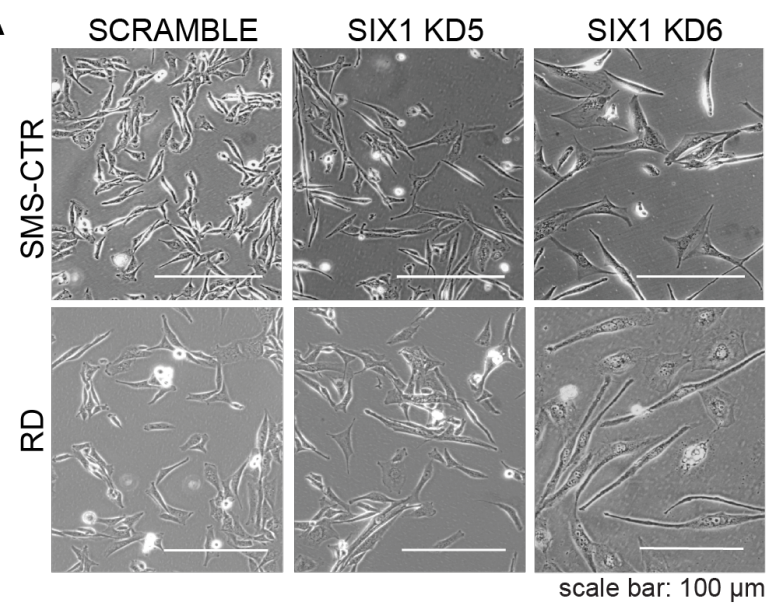

C

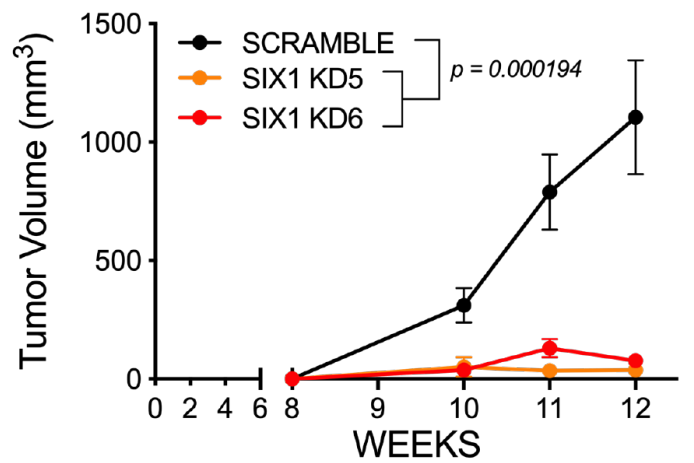

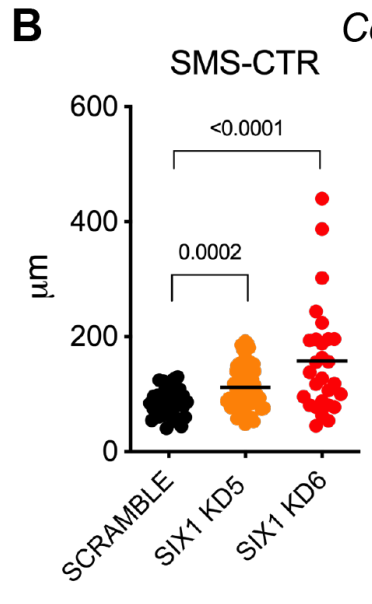

D
E

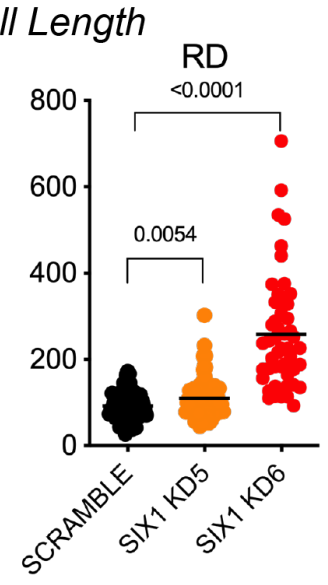

$\mathbf{F}$

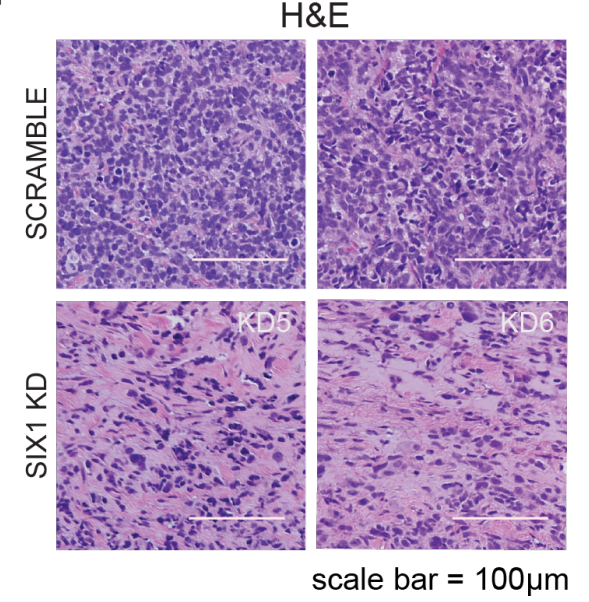

G
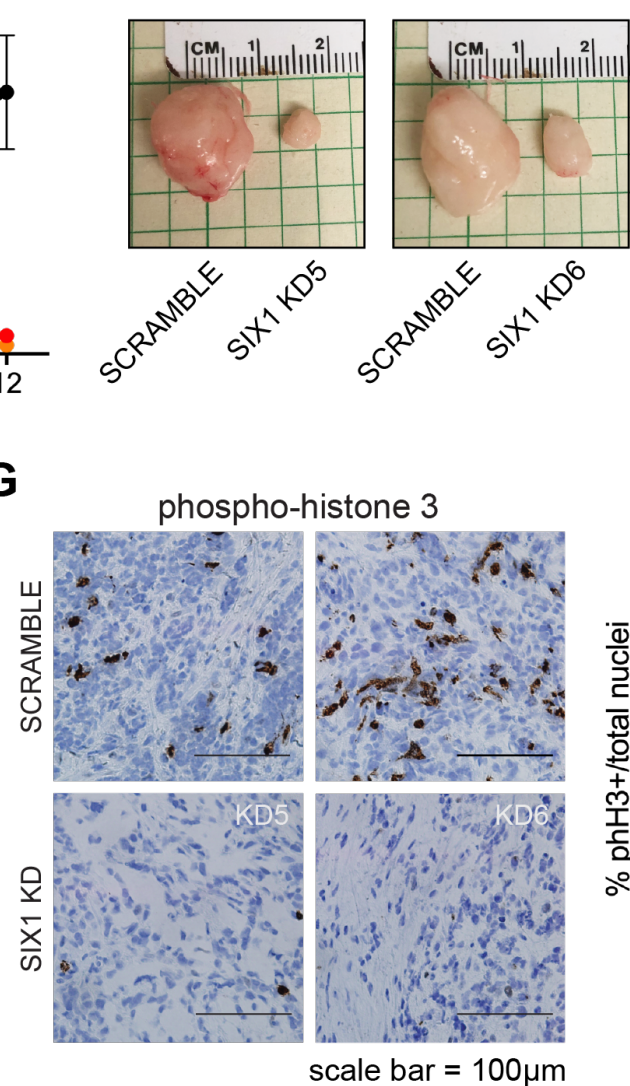
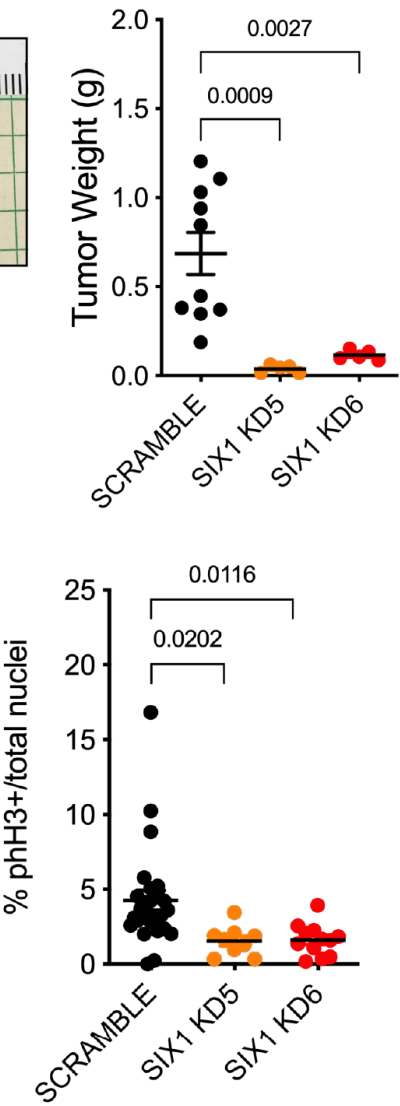
bioRxiv preprint doi: https://doi.org/10.1101/2021.04.25.439216; this version posted May 16, 2021. The copyright holder for this preprint (which was not certified by peer review) is the author/funder. All rights reserved. No reuse allowed without permission.

\section{Figure 4. SIX1 knockdown induces myogenic differentiation in RMS cells}

A

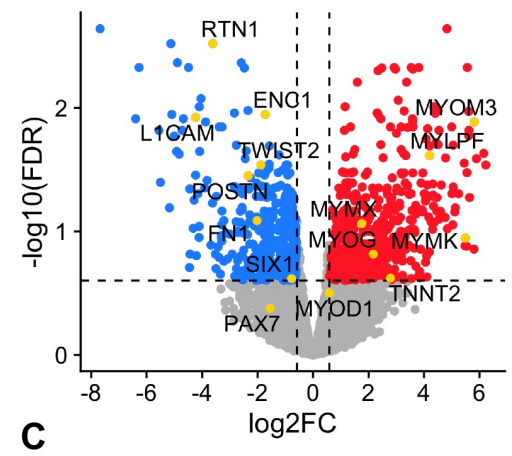

C

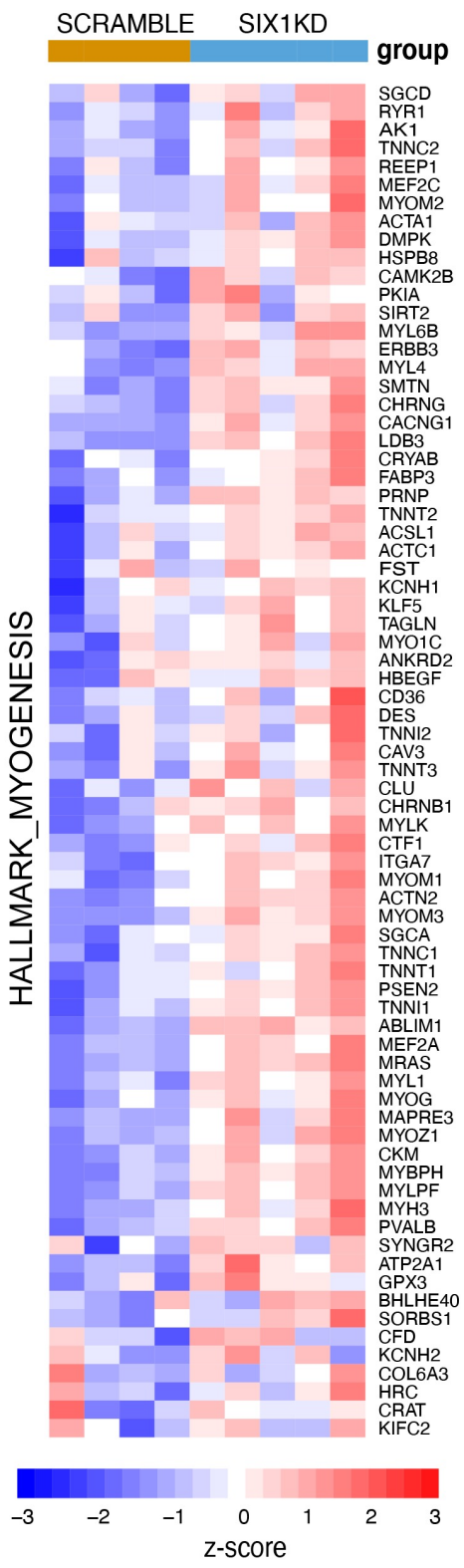

B
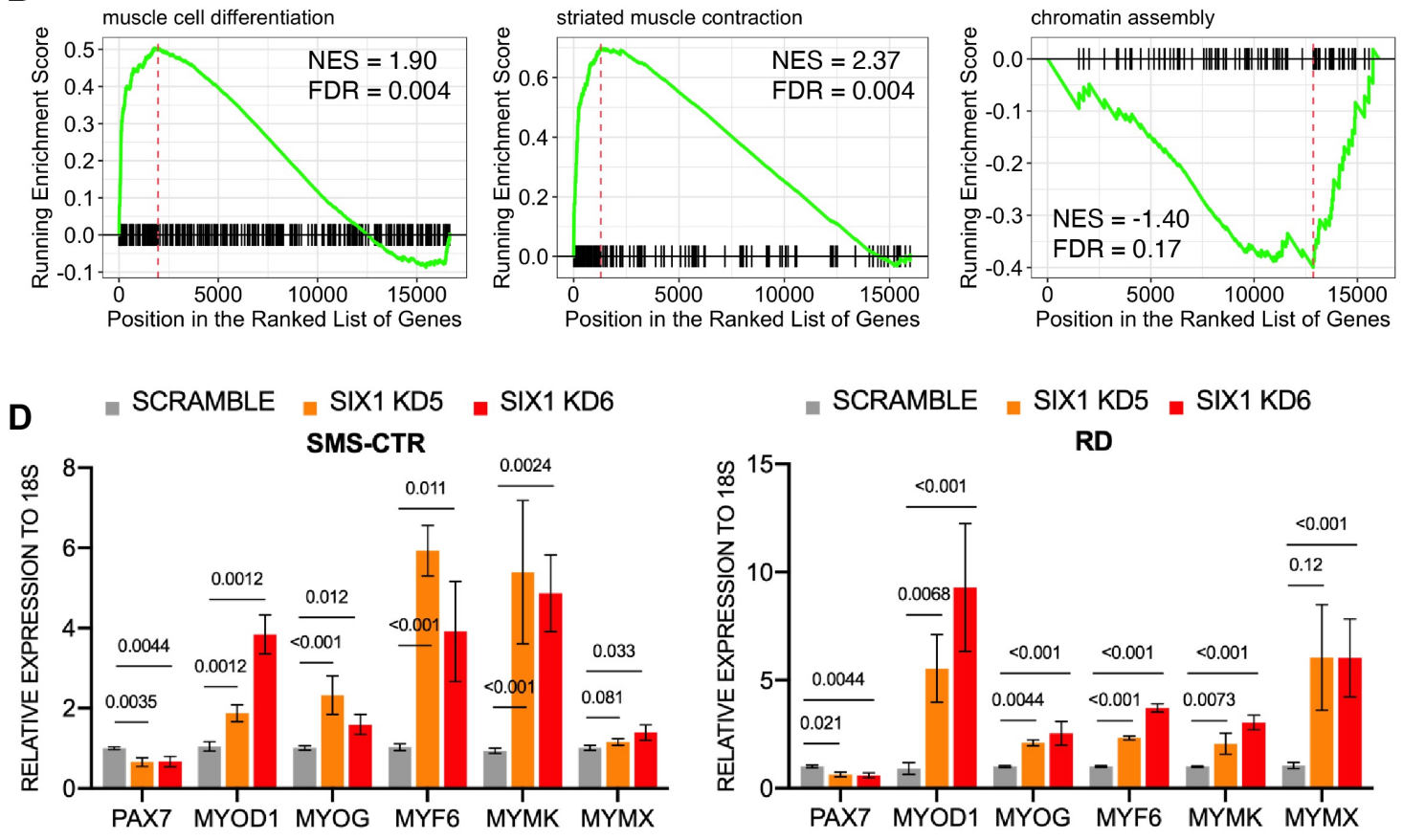

E
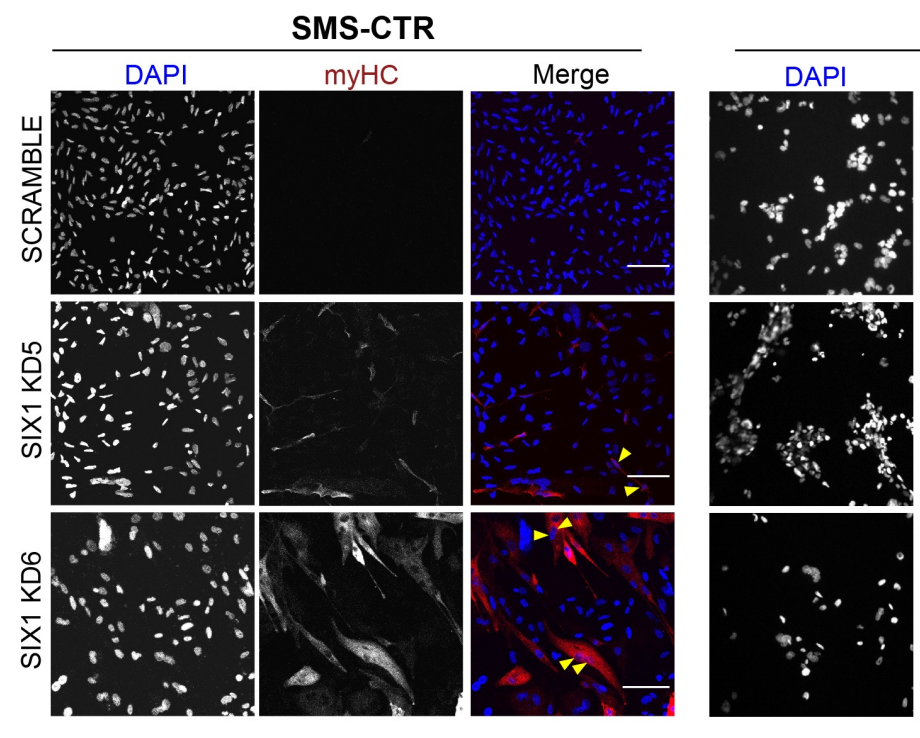

RD
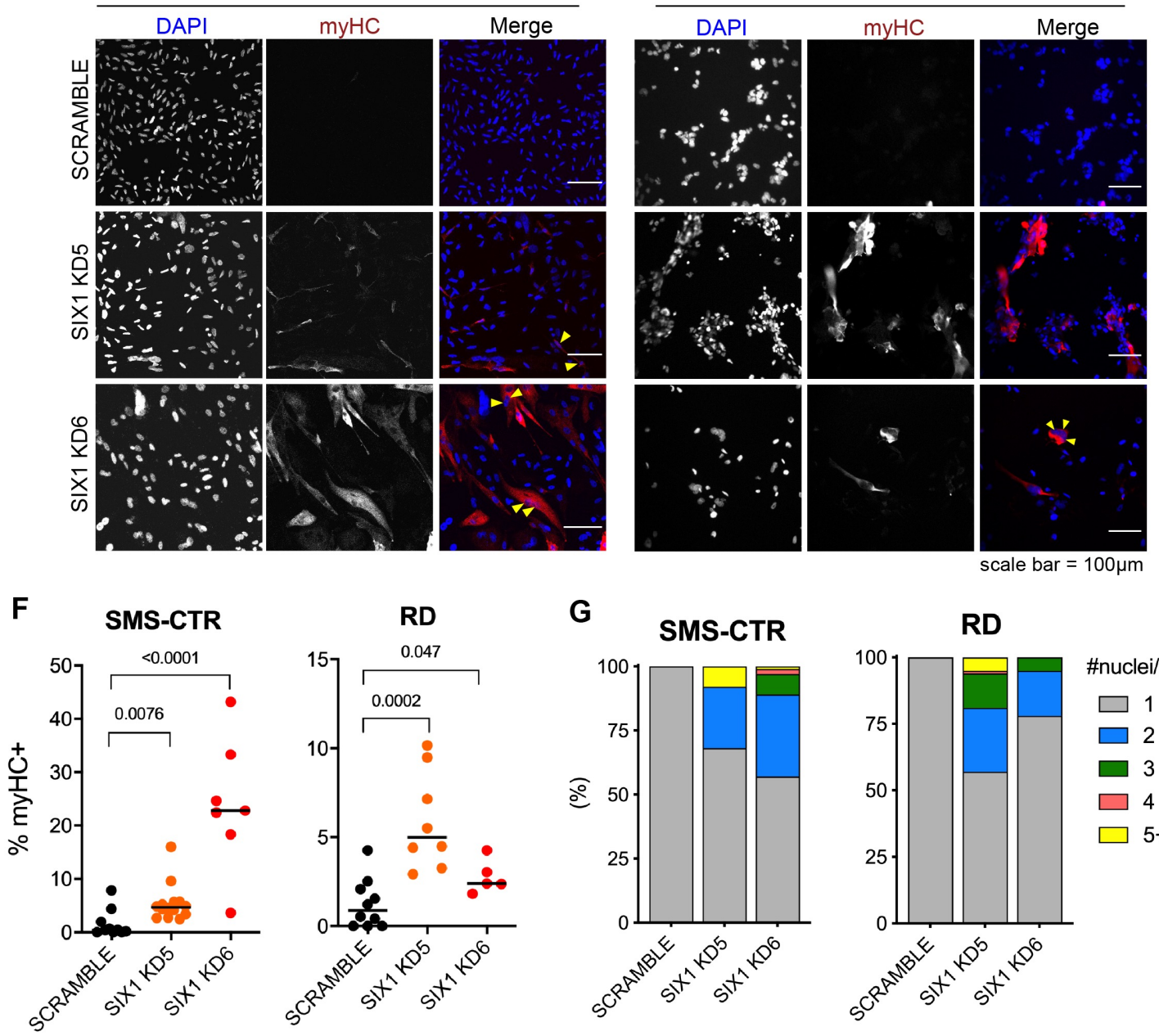

G
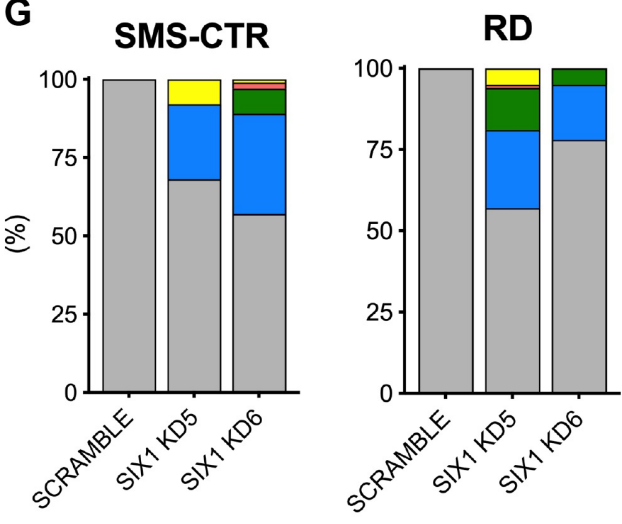

\#nuclei/myHC+

3

$\square 4$

$\square$ 5+ 
bioRxiv preprint doi: https://doi.org/10.1101/2021.04.25.439216; this version posted May 16, 2021. The copyright holder for this preprint (which was not certified by peer review) is the author/funder. All rights reserved. No reuse allowed without permission.

\section{Figure 5. SIX1 globally regulates both stem/oncogenic and myogenic differentiation genes through fine-tuning of super- enhancer activity}

A

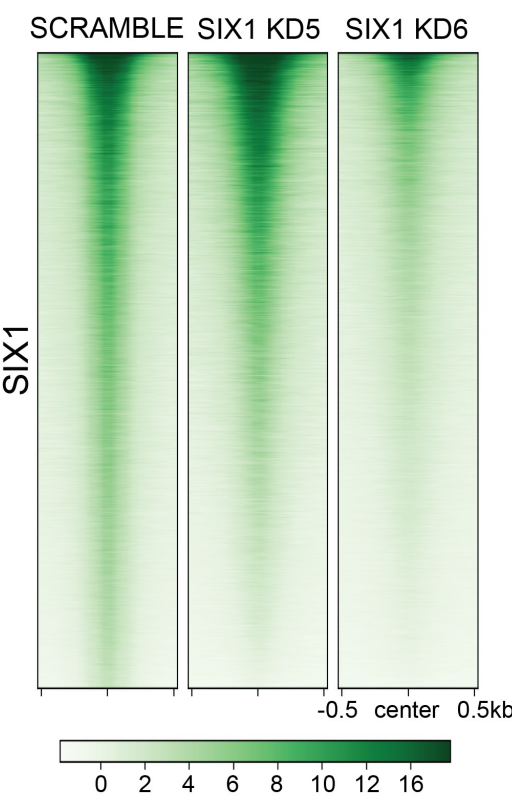

E

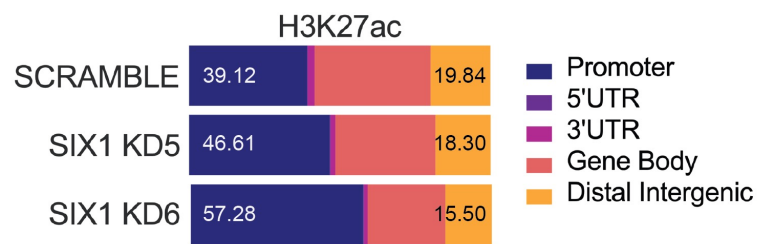

G

\section{H3K27ac-defined Super Enhancers}

skeletal muscle organ development regulation of cell-matrix adhesion skeletal muscle tissue development negative regulation of leukocyte differentiation adherens junction organization negative regulation of hemopoiesis morphogenesis of a branching structure regulation of ossification kidney epithelium development muscle cell development

actin filament-based movement muscle filament slidin actin-myosin filament sliding actin-mediated cell contraction adherens junction organization substrate adhesion-dependent cell spreading regulation of cell-matrix adhesion positive regulation of cell-substrate adhesion cell-substrate junction assembly kidney epithelium development

C

\begin{tabular}{|c|c|c|}
\hline $\begin{array}{c}\text { MOTIF } \\
\end{array}$ & TF & $\begin{array}{l}(\%) \\
\text { TARGETS }\end{array}$ \\
\hline 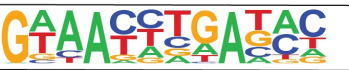 & SIX2 & 45.99 \\
\hline GGATCAGTGTIAC & SIX1 & 22.05 \\
\hline GGATGACTCATC: & FRA2 & 21.62 \\
\hline 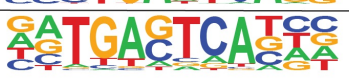 & FOSL2 & 17.07 \\
\hline
\end{tabular}

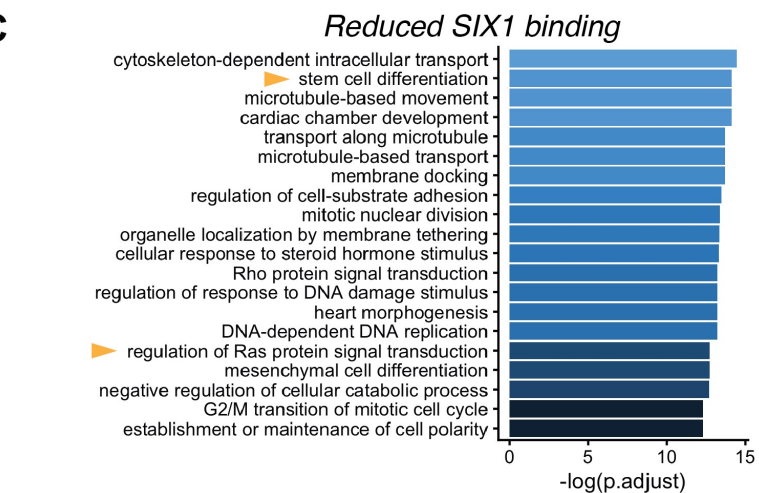

$\mathbf{F}$

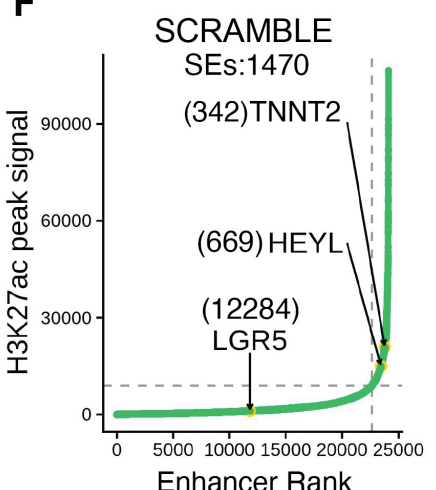

H
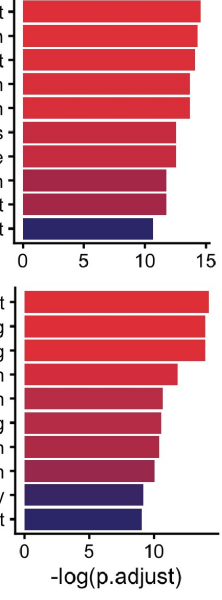

D

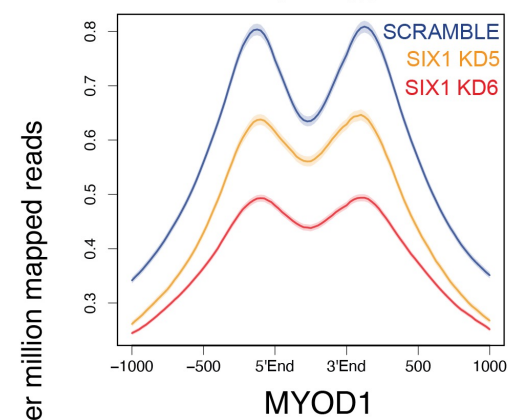

H3K27ac

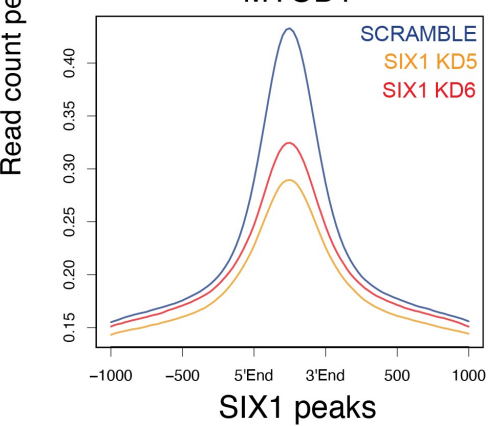

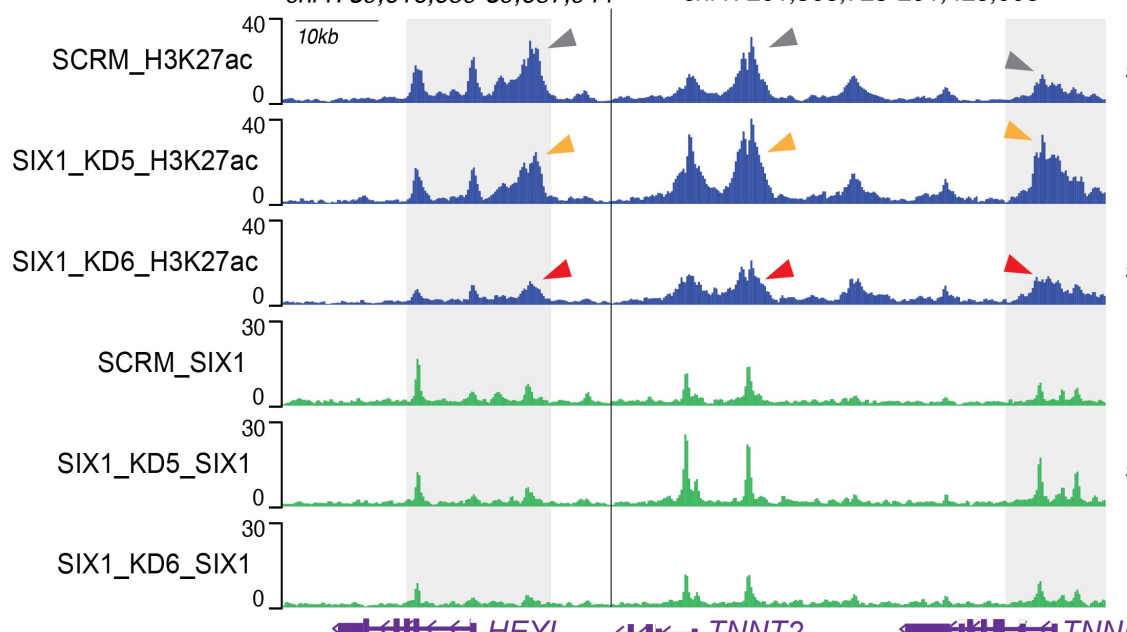

SIX1 KD5

SEs:1452
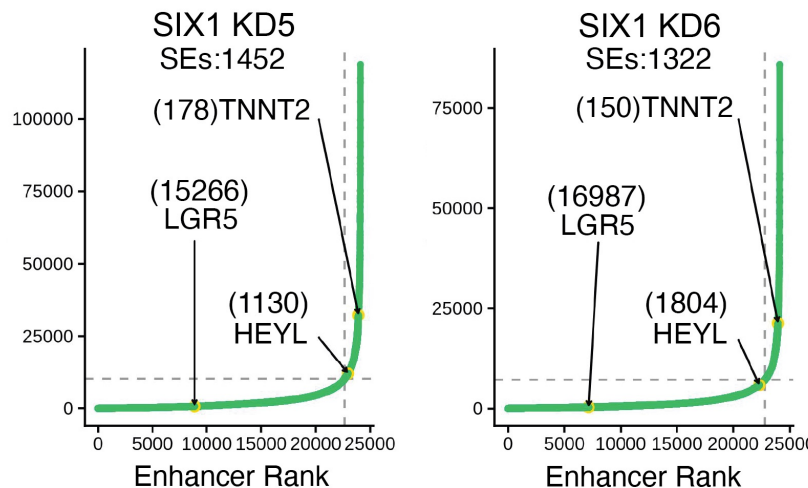

Enhancer Rank

$\leadsto H E Y L$

$\leftrightarrow \leftrightarrow$ TNNT2

-HHL-TNNI1

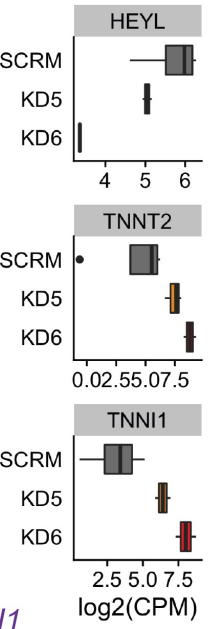


bioRxiv preprint doi: https://doi.org/10.1101/2021.04.25.439216; this version posted May 16, 2021. The copyright holder for this preprint (which was not certified by peer review) is the author/funder. All rights reserved. No reuse allowed without permission.

\section{Figure 6. SIX1 loss alters MYOD1 occupancy at muscle differentiation and stem/oncogenic loci}

A

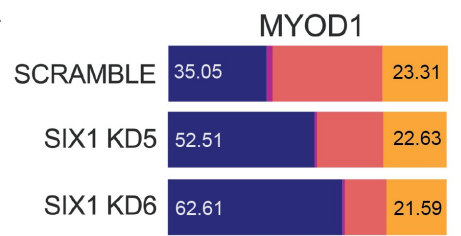

B

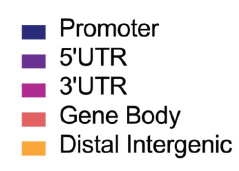

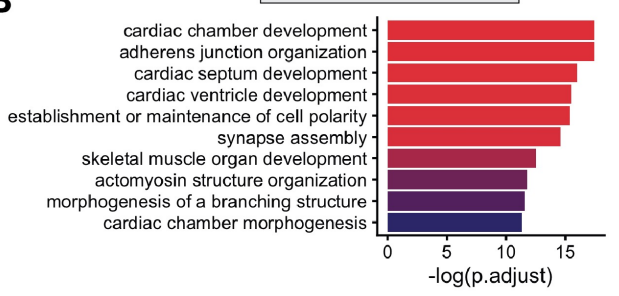

chr19:15, 195,247-15,225,688

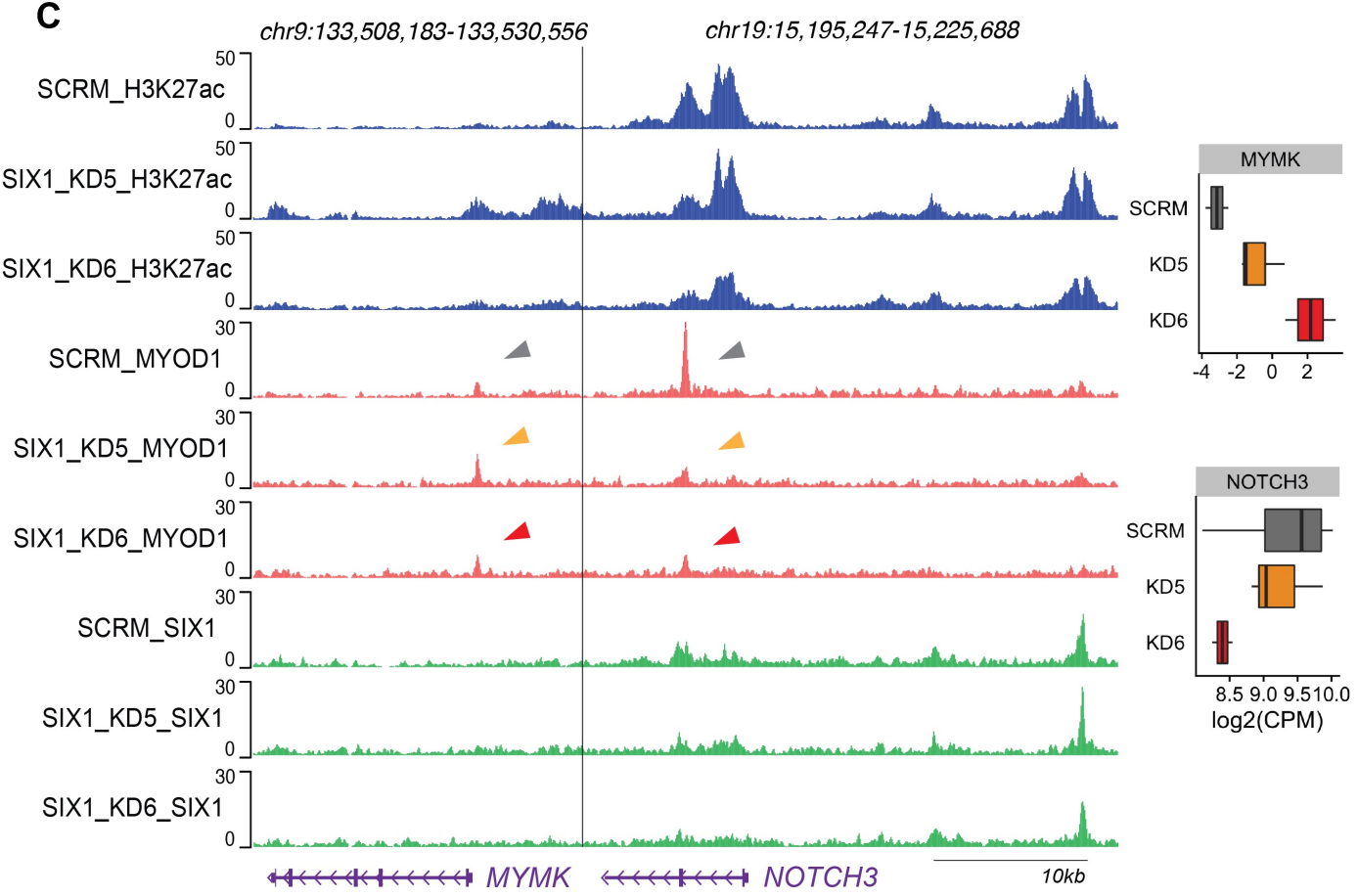

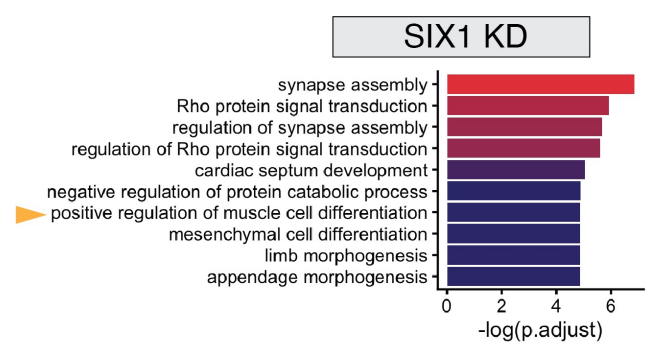

D

MYOD1 CUT\&RUN

. SCRAMBLE \#SIX1 KD5
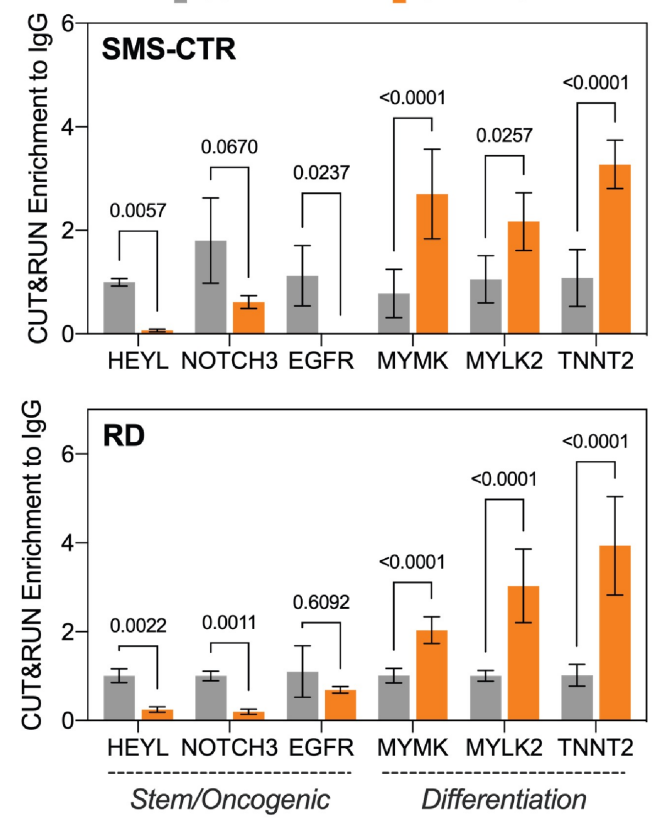
bioRxiv preprint doi: https://doi.org/10.1101/2021.04.25.439216; this version posted May 16, 2021. The copyright holder for this preprint (which was not certified by peer review) is the author/funder. All rights reserved. No reuse allowed without permission.

\section{Figure 7. SIX1 expression in RMS patients is inversely correlated with a mvotube qene sianature}

A

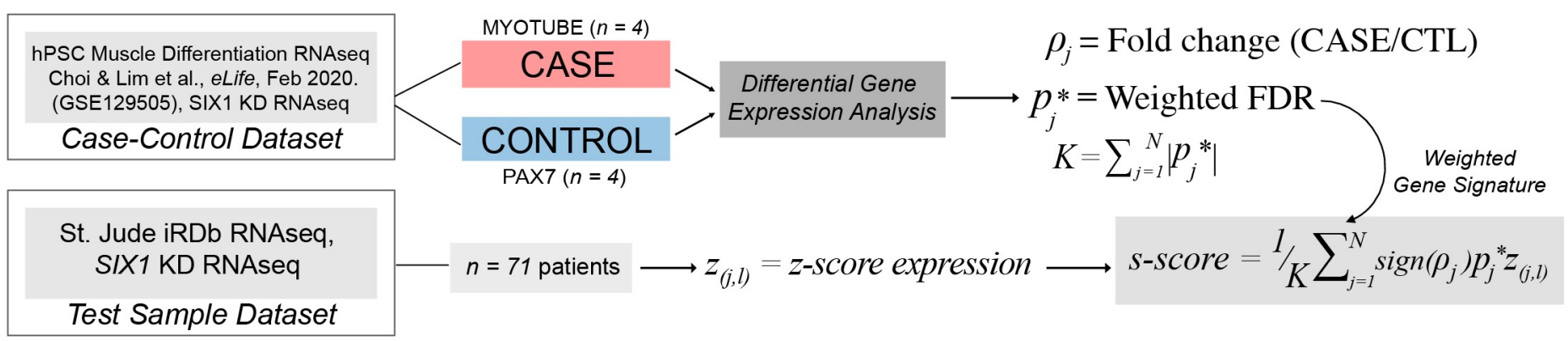

B

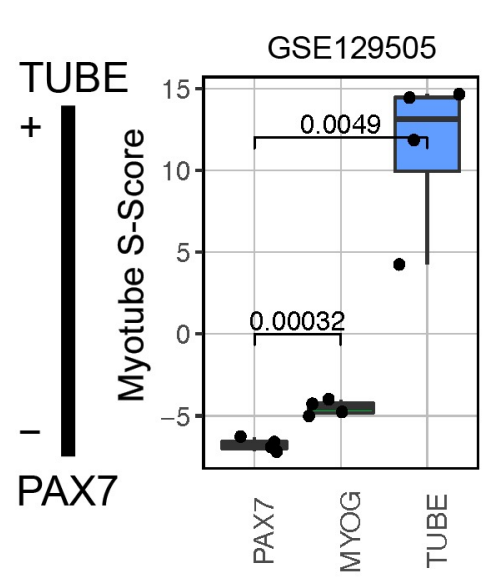

C

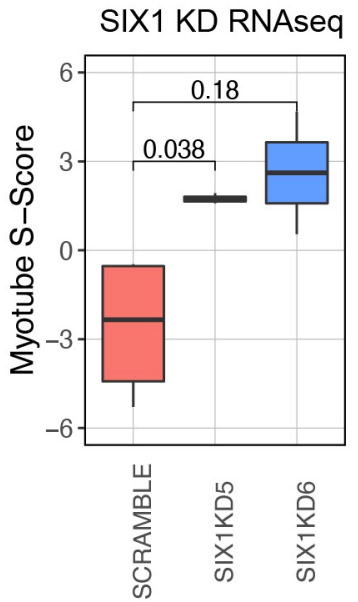

D St Jude iRDb

F St Jude iRDb
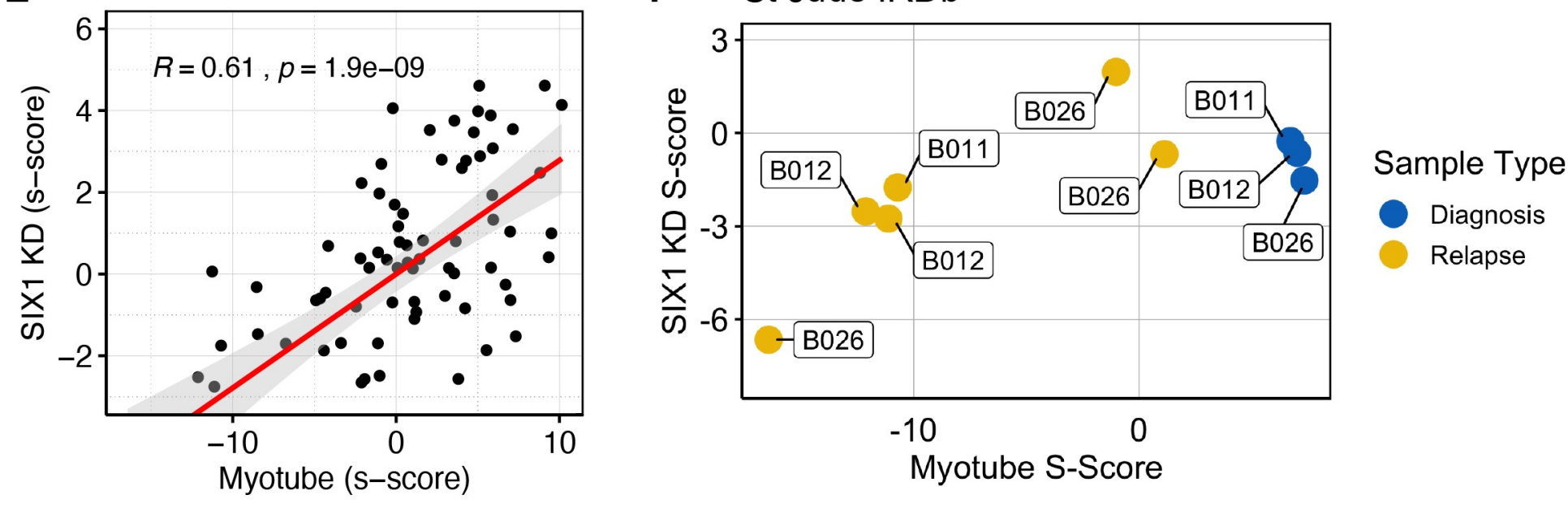


\section{SIX1: $\quad++++$}
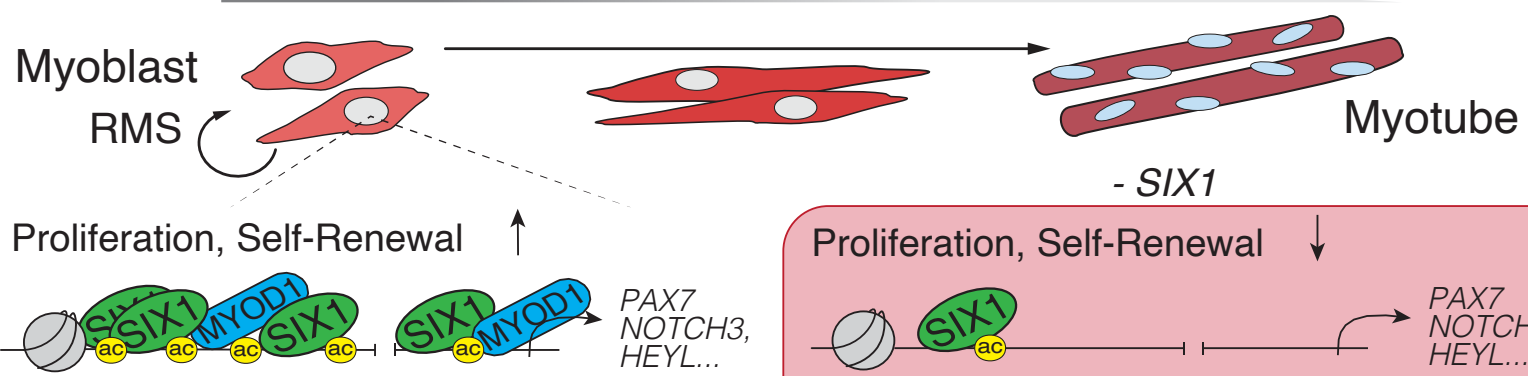

Proliferation, Self-Renewal $\downarrow$

Differentiation, Muscle Contraction

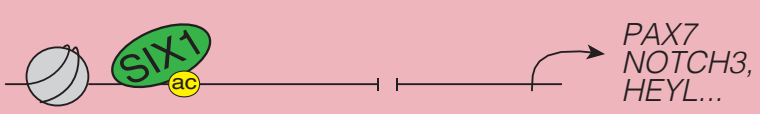

Differentiation, Muscle Contraction $\uparrow$
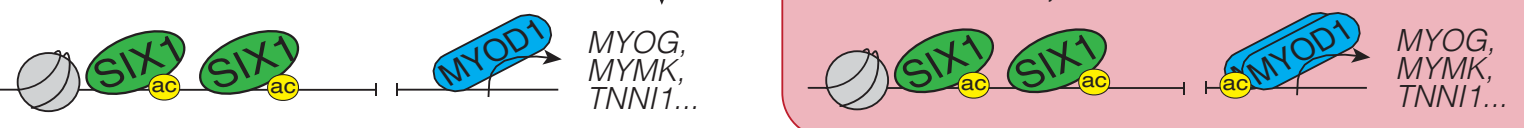